\title{
INVARIANTS AND SEPARATING MORPHISMS FOR ALGEBRAIC GROUP ACTIONS
}

\author{
EMILIE DUFRESNE AND HANSPETER KRAFT
}

\begin{abstract}
The first part of this paper is a refinement of Winkelmann's work on invariant rings and quotients of algebraic group actions on affine varieties, where we take a more geometric point of view. We show that the (algebraic) quotient $X / / G$ given by the possibly not finitely generated ring of invariants is "almost" an algebraic variety, and that the quotient morphism $\pi: X \rightarrow X / / G$ has a number of nice properties. One of the main difficulties comes from the fact that the quotient morphism is not necessarily surjective.

These general results are then refined for actions of the additive group $\mathbb{G}_{a}$, where we can say much more. We get a rather explicit description of the socalled plinth variety and of the separating variety, which measures how much orbits are separated by invariants. The most complete results are obtained for representations. We also give a complete and detailed analysis of ROBERTS' famous example of a an action of $\mathbb{G}_{a}$ on 7-dimensional affine space with a non-finitely generated ring of invariants.
\end{abstract}

\section{INTRODUCTION}

In all classification problems invariants play an important rôle. They let one distinguish nonequivalent objects, characterize specific elements, or detect certain properties. For instance, the genus of a complex smooth projective curve $C$ determines the topology of the compact surface $C$, and the discriminant of a polynomial tells us whether it has multiple roots. But there are many other examples of important invariants, like the ALEXANDER-polynomial of a knot or the DEDEKIND $\zeta$-function of a number field.

In the algebraic setting where we work over an algebraically closed field $\mathbb{k}$, we can often reduce a classification problem to the following general situation. There is an algebraic variety $X$ representing the objects, and an algebraic group $G$ acting on $X$ such that two objects $x, y \in X$ are equivalent if and only if they belong to the same orbit under $G$. In this case the classification problem amounts to describing the orbit space $X / G$. Clearly, $X / G$ inherits some properties from $X$ : it has a topology and the (continuous) functions on $X / G$ correspond to the (continuous) $G$-invariant functions on $X$. Of course, we would like to see $X / G$ again as an algebraic variety, but this cannot work in general, because $X$ usually contains nonclosed orbits, and so $X / G$ contains nonclosed points.

If $X$ is an affine variety with coordinate $\operatorname{ring} \mathcal{O}(X)$, we can look at the subalgebra $\mathcal{O}(X)^{G} \subset \mathcal{O}(X)$ of $G$-invariant functions and consider the morphism

$$
\pi_{X}: X \rightarrow X / / G:=\operatorname{Spec} \mathcal{O}(X)^{G}
$$

Date: Wednesday $14^{\text {th }}$ January, 2015.

The authors were partially supported by the SNF (Schweizerischer Nationalfonds). 
induced by the inclusion. It is a categorical quotient in the category of affine $\mathbb{k}$ schemes, and has the usual universal property: Every $G$-invariant morphism $X \rightarrow Y$ factors uniquely through $\pi_{X}$. In some sense this is the best schematic approximation to the orbit space. We will say that $X / / G$ is the quotient scheme and $\pi_{X}: X \rightarrow X / / G$ the quotient morphism or shortly the quotient.

If $G$ is reductive, then $\mathcal{O}(X)^{G}$ is finitely generated and so $X / / G$ is an affine variety. Moreover, $\pi_{X}$ has some nice properties ([MFK94, chap. 1.2 Theorem 1.1]):

- $\pi_{X}$ is $G$-closed: If $Z \subset X$ is $G$-stable and closed, then $\pi_{X}(Z)$ is closed.

- $\pi_{X}$ is $G$-separating: If $Z, Z^{\prime} \subset X$ are disjoint $G$-stable closed subsets, then $\pi_{X}(Z) \cap \pi_{X}\left(Z^{\prime}\right)=\emptyset$.

In particular, $\pi_{X}$ is surjective and every fiber contains a unique closed orbit. Thus the variety $X / / G$ classifies the closed orbits in $X$. In good situations, the general orbits are closed, and so, at least generically, $X / / G$ is the orbit space.

If $G$ is not reductive, then all this fails to be true. In particular, the invariant ring might not be finitely generated and so the quotient $X / / G$ is not an algebraic variety, and the quotient morphism $\pi_{X}$ is usually not surjective. The fact that $X / / G$ is not of finite type is considered to be the main difficulty in handling non-reductive groups. We think that the non-surjectivity of $\pi_{X}$ is even a more serious problem.

One of the aims of this paper is to show that the quotient $X / / G$ as a $\mathbb{k}$-scheme is "almost algebraic" in the following sense. An open subset $U$ of a $\mathbb{k}$-scheme is called an algebraic variety or shortly algebraic if $U$, as a reduced scheme, is separated and of finite type. (The separatedness is generally not an issue here, because we are working with affine schemes.) Then we show that $X / / G$ contains large open algebraic subsets and that it shares many properties with algebraic varieties. This is explained in sections 2 and 4 which are inspired by WinKELMANN's work [Win03]. For example, if the base field is uncountable, then $X / / G$ is a JACOBson scheme which implies that the ZARISKI topology on $X / / G$ is determined by the ZARISKI topology on the $\mathbb{k}$-rational points of $X / / G$.

To have an idea of our approach and our results let us give a geometric interpretation of RoBERTs famous example of an action of the additive group $\mathbb{G}_{a}=(\mathbb{k},+)$ on $\mathbb{A}^{7}$ with a non-finitely generated ring of invariants. The details are given in the last section 9. Let $\pi: \mathbb{A}^{7} \rightarrow \mathbb{A}^{7} / / \mathbb{G}_{a}$ be the quotient.

(a) The fixed point set $F:=\left(\mathbb{A}^{7}\right)^{\mathbb{G}_{a}} \simeq \mathbb{A}^{4}$ is mapped under $\pi$ to a single point $\pi(0) \in \mathbb{A}^{7} / / \mathbb{G}_{a} ;$

(b) The complement $\mathbb{A}_{b d}^{7}:=\mathbb{A}^{7} \backslash F$ is a principal $\mathbb{G}_{a}$-bundle over its image $\pi\left(\mathbb{A}_{b d}^{7}\right) \subset X / / \mathbb{G}_{a}$ which is an open algebraic subset and contains every open algebraic subset $U$ of $\mathbb{A}^{7} / / \mathbb{G}_{a}$;

(c) The image of $\pi$ is $\pi\left(\mathbb{A}^{7}\right)=\pi\left(\mathbb{A}_{b d}^{7}\right) \cup\{\pi(0)\}$.

(d) The complement $\left(\mathbb{A}^{7} / / \mathbb{G}_{a}\right) \backslash \pi\left(\mathbb{A}_{b d}^{7}\right)$ is isomorphic to $\mathbb{A}^{3}$.

An important new feature is the concept of a separating morphism $\varphi: X \rightarrow Y$ where $Y$ is an algebraic variety (cf. [DK02, section 2.3]). This means that $\varphi$ is $G$ invariant and separates the same orbits as $\pi_{X}$ does. Such morphisms always exist even when the invariants are not finitely generated, but finding a "nice" separating morphism is usually a difficult task. For RoBERTs example we get the following.

(e) There exists a separating morphism $\varphi: \mathbb{A}^{7} \rightarrow \mathbb{A}^{9}$ such that $Y:=\overline{\varphi\left(\mathbb{A}^{7}\right)}$ is normal of dimension 6 . 
(f) The induced map $\bar{\varphi}: \mathbb{A}^{7} / / \mathbb{G}_{a} \rightarrow Y$ is injective. It defines a homeomorphism $\pi\left(\mathbb{A}^{7}\right) \rightarrow \varphi\left(\mathbb{A}^{7}\right)$ and an isomorphism $\pi\left(\mathbb{A}_{b d}^{7}\right) \stackrel{\sim}{\rightarrow} \varphi\left(\mathbb{A}_{b d}^{7}\right)$.

(g) $H:=Y \backslash \varphi\left(\mathbb{A}_{b d}^{7}\right)$ is a hypersurface in $Y$, and $\mathcal{O}\left(\mathbb{A}^{7}\right)^{\mathbb{G}_{a}}=\mathcal{O}(Y \backslash H)$.

Another important concept is the separating variety which measures how much the invariants separate the orbits. It is defined as the reduced fiber product $\mathcal{S}_{X}:=$ $X \times_{X / / G} X$ and contains the closure of the graph $\Gamma_{X}:=\{(g x, x) \mid g \in G, x \in X\}$. If a general fiber of the quotient map is an orbit and if $G$ is connected, then $\overline{\Gamma_{X}}$ is an irreducible component of the separating variety. But even in nice situations, the separating variety may have additional components. In general, the meaning of these other components is not yet well understood, except for some special cases (see below). For RoBERTs' example we find the following.

(h) The separating variety has two irreducible components: $\mathcal{S}_{\mathbb{A}^{7}}=\overline{\Gamma_{\mathbb{A}^{7}}} \cup F \times F$, both of dimension 8.

The most complete results are obtained for actions of the additive group $\mathbb{G}_{a}$, in particular for representations of $\mathbb{G}_{a}$ (sections $5-7$ ). This part of our work was inspired by certain calculations done by ELMER and KoHLS in [EK12]. An important tool here is the geometric interpretation of the zero set $\mathcal{P}_{X}$ of the plinth ideal (Definition 5.2). If $X$ is factorial, then $X \backslash \mathcal{P}_{X}$ is equal to the open set $X_{b d}$ where $X$ is locally a $\mathbb{G}_{a}$-bundle. In section 8 we generalize some of the results for representations of $\mathbb{G}_{a}$ to $\mathbb{G}_{a}$-actions induced by actions of $\mathrm{SL}_{2}$.

To prepare the reader for the difficulties in working with non-finitely generated algebras we describe an easy example in section 3.

\section{General Setup And Notation}

Invariants. Our base field $\mathbb{k}$ is algebraically closed. In the second part, starting with section 5 , we study $\mathbb{G}_{a}$-actions and will assume that char $\mathbb{k}=0$. Since we have to deal with non-finitely generated rings of invariants, we will work in the category of reduced $\mathbb{k}$-schemes $Z$. However, from the geometric point of view we are mainly interested in the $\mathbb{k}$-rational points of $Z$ which will denote by $Z(\mathbb{k})$. In this setting, a variety $Z$ is a reduced separated $\mathbb{k}$-scheme of finite type, and in this case we will often confuse the scheme $Z$ with its $\mathbb{k}$-rational points $Z(\mathbb{k})$.

Throughout this paper, we let $X$ be a normal affine variety and $G$ an algebraic group acting on $X$. We denote by $\mathcal{O}(X)$ the $\mathbb{k}$-algebra of regular functions on $X$ and by $\mathcal{O}(X)^{G} \subset \mathcal{O}(X)$ the subalgebra of $G$-invariant functions. The quotient is defined to be the affine $\mathbb{k}$-scheme

$$
X / / G:=\operatorname{Spec} \mathcal{O}(X)^{G} .
$$

If the base field $\mathbb{k}$ is uncountable, a famous result of KRULL's implies that $X / / G$ is a JACOBSOn scheme, i.e., $\mathcal{O}(X)^{G}$ is a JACOBSOn ring ([Kru51]). This means that every radical ideal of $\mathcal{O}(X)^{G}$ is the intersection of maximal ideals. Moreover, every closed point of $X$ is $\mathbb{k}$-rational in this case, since $\mathcal{O}(X)^{G}$ is contained in a finitely generated $\mathbb{k}$-algebra. It follows that the ZARISKI-topology on $X / / G$ is completely determined by the ZARISKI-topology on the $\mathbb{k}$-rational points $(X / / G)(\mathbb{k})$. This allows to work with $\mathbb{k}$-rational points which are the only interesting objects from a geometric point of view, as mentioned above.

Remark 2.1. If the $\mathbb{k}$-algebra $R$ is not a JACOBSON ring, then there is a prime ideal $\mathfrak{p} \subset R$ which is not the intersection of the maximal ideal containing $\mathfrak{p}$. In geometric 
terms this means the following. Denote by $Z \subset$ Spec $R$ the closed subscheme defined by $\mathfrak{p}$, and let $Z_{c l} \subset Z$ be the subset of closed points. Then the closure $\overline{Z_{c l}}$ in Spec $R$ is strictly contained in $Z$.

Quotient morphism. The inclusion $\mathcal{O}(X)^{G} \hookrightarrow \mathcal{O}(X)$ defines the quotient morphism

$$
\pi=\pi_{X}: X \rightarrow X / / G
$$

Although $\mathcal{O}(X)^{G}$ might not be finitely generated, hence $X / / G$ is not of finite type, we will see that the quotient $X / / G$ contains large open subschemes which are varieties. For this we need the following result due to DERKSEN and KEMPER [DK08, Propositions 2.7 and 2.9].

Proposition 2.2. Let $R$ be a $\mathbb{k}$-algebra. Define

$$
\mathfrak{f}_{R}:=\left\{f \in R \mid R_{f} \text { is finitely generated }\right\} \cup\{0\} .
$$

Then $\mathfrak{f}_{R}$ is a radical ideal of $R$. If $R$ is contained in a finitely generated $\mathbb{k}$-domain, then $\mathfrak{f}_{R} \neq(0)$.

The ideal $\mathfrak{f}_{R}$ will be called the finite generation ideal.

Remark 2.3. The open subset $\operatorname{Spec} R \backslash \mathcal{V}\left(\mathfrak{f}_{R}\right) \subset \operatorname{Spec} R$ is the union of all open subsets $U \subset \operatorname{Spec} R$ which are algebraic. In fact, each such $U$ is a finite union of open affine varieties $U_{i}$, and each $U_{i}$ is a finite union of some $(\operatorname{Spec} R)_{f_{j}}$. We will denote the complement $\operatorname{Spec} R \backslash \mathcal{V}\left(\mathfrak{f}_{R}\right)$ by $(\operatorname{Spec} R)_{\text {alg }}$ :

$$
(\operatorname{Spec} R)_{\text {alg }}:=\operatorname{Spec} R \backslash \mathcal{V}\left(\mathfrak{f}_{R}\right)=\bigcup_{\substack{U \subset \operatorname{Spec} R \\ U \text { open algebraic }}} U \subseteq \operatorname{Spec} R .
$$

Note that $(\operatorname{Spec} R)_{a l g}$ is itself a variety if and only if $\mathfrak{f}_{R}$ is the radical of a finitely generated ideal. On the other hand, $(\operatorname{Spec} R)_{a l g}$ is always JACOBSON and its closed points coincide with its $\mathbb{k}$-rational points.

Definition 2.4. Let $Z=\operatorname{Spec} R$ be an affine $\mathbb{k}$-scheme. If $A \subset Z$ is a closed subset we define $I(A) \subset R$ to be the (radical) ideal of functions vanishing on $A$.

(a) $\operatorname{dim} Z:=\operatorname{Kdim} R$ is the KRULL-dimension of $R$.

(b) If $Z$ is reduced and irreducible, i.e., if $R$ is a domain, then $\mathbb{k}(Z):=Q(R)$ denotes the field of fractions of $R$.

(c) If $R$ is a domain, then $\operatorname{tdeg}_{\mathbb{k}} R:=\operatorname{tdeg}_{\mathbb{k}} Q(R)$ is the transcendence degree of the field extension $Q(R) / \mathbb{k}$.

(d) If $A \subset Z$ is closed, then $\operatorname{codim}_{Z} A:=\min \{$ ht $\mathfrak{p} \mid \mathfrak{p} \supset I(A), \mathfrak{p}$ prime $\}$ where ht $\mathfrak{p}$ is the height of the prime ideal $\mathfrak{p}$.

As an example, we will see later in Theorem $4.3(\mathrm{a})$ that the quotient $X / / G$ is always finite dimensional, and that $\operatorname{dim} X / / G=\operatorname{tdeg}_{\mathbb{k}} \mathcal{O}(X)^{G}$.

Algebraic varieties. Assume that $Z=\operatorname{Spec} R$ is a variety. Then $Z=\bigcup_{i} Z_{i}$ is a finite union of irreducible closed subsets, and $\operatorname{dim} Z=\max _{i}\left\{\operatorname{dim} Z_{i}\right\}$. Moreover, if $Z$ is irreducible, then $\operatorname{dim} Z=\operatorname{tdeg}_{\mathbb{k}} R$, and for every irreducible closed subset $A \subset Z$ we have $\operatorname{dim} A+\operatorname{codim}_{Z} A=\operatorname{dim} Z$.

Finally, if $\varphi: Z \rightarrow Y$ is a morphism where $Y$ is an arbitrary reduced $\mathbb{k}$-scheme, and if $A \subset Z$ is a closed subscheme, then $\varphi(A(\mathbb{k}))$ is dense in $\varphi(A) \subset Y$. As mentioned before, this last statement holds more generally if $Z$ is a JACOBSON scheme. 


\section{A First Example}

Let us discuss an interesting example. While it does not quite fit in our settingit does not arise from a quotient of an algebraic group action on a normal affine variety - it has a similar behavior.

Consider the graded subring $R:=\mathbb{k}\left[x, x y, x y^{2}, x y^{3}, \ldots\right] \subset \mathbb{k}[x, y]$ generated by the monomials $x y^{k}, k=0,1, \ldots$, and set $Z:=\operatorname{Spec} R$.

(a) The finite generation ideal $\mathfrak{f}_{R}$ of $R$ is equal to the homogeneous maximal ideal $\mathfrak{m}_{0}=\left(x, x y, x y^{2}, \ldots\right)$, and $\mathfrak{m}_{0}=\sqrt{x R}$.

(b) We have $Z \backslash\left\{\mathfrak{m}_{0}\right\}=Z_{x}$, and this is an affine algebraic variety with coordinate ring $\mathbb{k}\left[x, x^{-1}, y\right]$

Now consider the morphism $\pi: \mathbb{A}^{2} \rightarrow Z$ given by the inclusion $R \subset \mathbb{k}[x, y]$. (This morphism plays the role of the quotient morphism.)

(c) $\pi: \mathbb{A}^{2} \rightarrow Z$ is surjective and induces an isomorphism $\left(\mathbb{A}^{2}\right)_{x} \stackrel{\sim}{\rightarrow} Z_{x}$.

(d) $\pi: \mathbb{A}^{2} \rightarrow Z$ is a closed morphism.

Finally, we consider the affine morphism $\varphi: \mathbb{A}^{2} \rightarrow \mathbb{A}^{2}$ given by $(x, y) \mapsto(x, x y)$. (This morphism plays the role of a separating morphism.)

(e) $\varphi$ factors through $\pi$

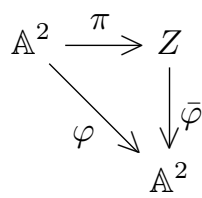

and $\bar{\varphi}$ is injective. Hence $\varphi$ separates the same points of $\mathbb{A}^{2}$ as $\pi$ does.

(f) $\bar{\varphi}$ induces a homeomorphism $Z \rightarrow \varphi\left(\mathbb{A}^{2}\right)=\mathbb{A}_{y}^{2} \cup\{0\}$.

The proofs are not difficult and are left to the reader. They are based on the following lemma.

Lemma 3.1. (a) We have $R=\mathbb{k} \oplus \mathfrak{m}_{0}$ where $\mathfrak{m}_{0}=x \mathbb{k}[x, y]=\left(x, x y, x y^{2}, \ldots\right)$ is the homogeneous maximal ideal of $R$.

(b) Let $f \in \mathbb{k}[x, y]$. Then

$$
f \mathbb{k}[x, y] \cap R= \begin{cases}f \mathbb{k}[x, y] & \text { if } f \in \mathfrak{m}_{0} ; \\ f R & \text { if } f \in R \backslash \mathfrak{m}_{0} ; \\ (x f) R & \text { if } f \notin R .\end{cases}
$$

\section{Separating Morphisms}

Separation. The so-called separation property will play an important role in this paper. The notion goes back to DERKSEN and KEMPER [DK02, section 2.3.2], and is also implicit in the work of WinKELMANN [Win03, Lemma 7].

Definition 4.1. Let $X$ be an affine $G$-variety. A $G$-invariant morphism $\varphi: X \rightarrow Y$ where $Y$ is an affine variety is a separating morphism if it satisfies the following Separation Property:

(SP) If $x, x^{\prime} \in X(\mathbb{k})$ are separated by an invariant $f \in \mathcal{O}(X)^{G}$, i.e., if $f(x) \neq$ $f\left(x^{\prime}\right)$, then $\varphi(x) \neq \varphi\left(x^{\prime}\right)$. 
Remark 4.2. If char $\mathbb{k}=0$, then the separation property (SP) implies that $\varphi^{*}$ induces an isomorphism $\mathbb{k}(\overline{\varphi(X)}) \stackrel{\sim}{\rightarrow} \mathbb{k}(X / / G)$. If char $\mathbb{k}>0$, we say that $\varphi$ is strongly separating if $\varphi$ is separating and induces an isomorphism $\mathbb{k}(\overline{\varphi(X)}) \stackrel{\sim}{\longrightarrow}$ $\mathbb{k}(X / / G)$.

It is shown in [DK02, Theorem 2.3.15] that separating morphisms always exist. In more algebraic terms this means that one can find a finitely generated separating subalgebra $R \subset \mathcal{O}(X)^{G}$, i.e., a subalgebra which separates the same $\mathbb{k}$-rational points of $X$ as the invariant functions. We can always add invariant functions to $R$, and thus assume that $R$ is normal and that $Q(R)=\mathbb{k}(X / / G)$, if necessary. Thus, a strongly separating morphism $\varphi: X \rightarrow Y$ with $Y$ normal always exists. A basic problem is to find a separating algebra with a small number of generators.

Main results. A $G$-invariant morphism $\varphi: X \rightarrow Y$ where $Y$ is an affine variety always factors through the quotient morphism $\pi: X \rightarrow X / / G$ :

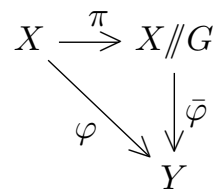

Then $\varphi$ is separating if and only if $\bar{\varphi}$ is injective on the image $\pi(X(\mathbb{k})) \subset(X / / G)(\mathbb{k})$ of the $\mathbb{k}$-rational points. In the paper [Win03] WINKELMANN studies this general set-up and proves a number of fundamental results, e.g. that every such invariant ring $\mathcal{O}(X)^{G}$ is the ring of global regular functions on a quasi-affine variety and vice versa. Some of his results are contained and extended in the following theorem, where we take a geometric point of view. From that point of view we are mainly interested in the images $\pi(X) \subset X / / G$ and $\varphi(X) \subset Y$ and how they are related to $(X / / G)_{\text {alg }}=X / / G \backslash \mathcal{V}\left(\mathfrak{f}_{X / / G}\right)$ where $\mathfrak{f}_{X / / G} \subset \mathcal{O}(X)^{G}$ denotes the finite generation ideal (Proposition 2.2 and Remark 2.3).

Theorem 4.3. Let $X$ be a normal affine variety with an action of an algebraic group $G$ and denote by $\pi: X \rightarrow X / / G$ the quotient morphism. Let $\varphi: X \rightarrow Y$ be a dominant separating morphism where $Y$ is a normal affine variety.

(a) If $A \subset X$ is an irreducible closed subset, then $\operatorname{dim} \overline{\pi(A)}=\operatorname{dim} \overline{\varphi(A)}$ and $\operatorname{codim}_{X / / G} \overline{\pi(A)}=\operatorname{codim}_{Y} \overline{\varphi(A)}$. In particular,

$$
\operatorname{dim} X / / G=\operatorname{dim} Y=\operatorname{tdeg}_{\mathbb{k}} \mathcal{O}(X)^{G} .
$$

(b) The map $\bar{\varphi}: X / / G \rightarrow Y$ induces a homeomorphism $\pi(X) \stackrel{\sim}{\rightarrow} \varphi(X)$.

(c) Let $C_{X}:=X / / G \backslash \pi(X)$ be the complement. Then $\operatorname{codim}_{X / / G} \overline{C_{X}}>1$.

(d) The complement $X / / G \backslash(X / / G)_{\text {alg }}$ has codimension $>1$ in $X / / G$.

Now assume that $\varphi$ is strongly separating, and let $C_{Y}:=Y \backslash \varphi(X)$ be the complement of the image of $\varphi$.

(e) $\bar{\varphi}^{*}$ induces an isomorphism $\mathcal{O}\left(Y \backslash \overline{C_{Y}}\right) \stackrel{\sim}{\longrightarrow} \mathcal{O}(X)^{G}$.

(f) $\bar{\varphi}^{-1}\left(Y \backslash \overline{C_{Y}}\right) \subseteq(X / / G)_{\text {alg }}$, and the induced map $\bar{\varphi}^{-1}\left(Y \backslash \overline{C_{Y}}\right) \stackrel{\sim}{\rightarrow} Y \backslash \overline{C_{Y}}$ is an isomorphism of varieties.

(g) $\bar{\varphi}$ induces an open immersion $(X / / G)_{a l g} \backslash \overline{C_{X}} \hookrightarrow Y$. 
Let us draw some diagrams. Suppose $\varphi$ is strongly separating. The statements (b) and (f) give

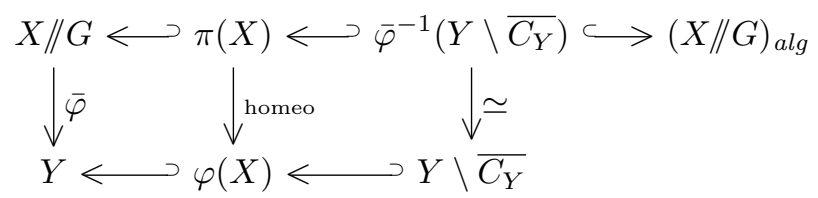

and from (e) we have

$$
\mathcal{O}(X / / G)=\mathcal{O}\left(\bar{\varphi}^{-1}\left(Y \backslash \overline{C_{Y}}\right) \simeq \mathcal{O}\left(Y \backslash \overline{C_{Y}}\right)\right.
$$

From the statements (b) and (g) we get

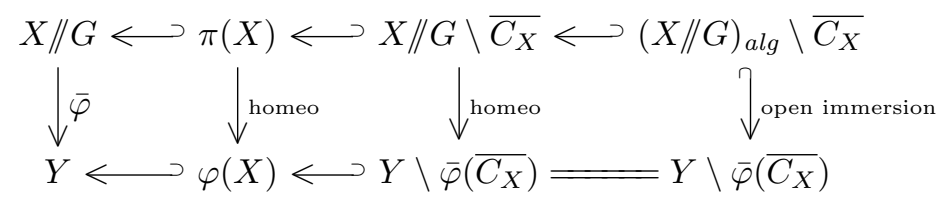

Corollary 4.4. Assume $\varphi: X \rightarrow Y$ is dominant and strongly separating with $Y$ normal. If $\pi(X) \supseteq(X / / G)_{\text {alg }}$, then $(X / / G)_{\text {alg }}$ is algebraic and $\bar{\varphi}$ induces an open immersion $(X / / G)_{\text {alg }} \hookrightarrow Y$.

Proof. Let $U \subseteq(X / / G)_{a l g}$ be an open algebraic subset. Then $\bar{\varphi}: U \rightarrow Y$ is injective and birational, hence an open immersion by ZARIskI's Main Theorem ([Mum99, III. $\S 9$, page 209]). Thus $\bar{\varphi}\left((X / / G)_{\text {alg }}\right) \subseteq Y$ is open and $\bar{\varphi}:(X / / G)_{\text {alg }} \stackrel{\sim}{\rightarrow} \bar{\varphi}\left((X / / G)_{\text {alg }}\right)$ is an isomorphism.

Corollary 4.5. Assume $\varphi: X \rightarrow Y$ is dominant and strongly separating. If $Y$ is factorial, then $\mathcal{O}(X)^{G}$ is finitely generated and $\bar{\varphi}: X / / G \rightarrow Y$ is an open immersion. In particular, $X / / G \simeq Y_{f}:=Y \backslash \mathcal{V}_{Y}(f)$ for a suitable $f \in \mathcal{O}(Y)$.

Proof. (a) If $\operatorname{codim}_{Y} \overline{C_{Y}}>1$, then $\mathcal{O}\left(Y \backslash \overline{C_{Y}}\right)=\mathcal{O}(Y)$ and so $\bar{\varphi}: X / / G \stackrel{\sim}{\longrightarrow} Y$ is an isomorphism, by Theorem 4.3(d).

(b) If $\operatorname{codim}_{Y} \overline{C_{Y}}=1$, then we have $\overline{C_{Y}}=\mathcal{V}_{Y}(f) \cup C$ where $f \in \mathcal{O}(Y)$ and $\operatorname{codim}_{Y} C>1$, because $Y$ is factorial. Hence $f, f^{-1} \in \mathcal{O}\left(Y \backslash \overline{C_{Y}}\right) \simeq \mathcal{O}(X / / G)$, and so $\bar{\varphi}(X / / G) \subseteq Y_{f}$. Thus we can replace $Y$ by $Y_{f}$ and get from (a) that $X / / G \stackrel{\sim}{\longrightarrow} Y_{f}$.

Corollary 4.6. If $V$ is a rational representation of $G$ and if $\varphi: V \rightarrow Y$ is dominant and strongly separating with $Y$ factorial, then $\bar{\varphi}: V / / G \stackrel{\sim}{\rightarrow} Y$ is an isomorphism.

Proof. This is clear from the previous corollary, because $\mathcal{O}(V)^{G}$ does not contain nonconstant invertible functions.

Remark 4.7. In the case where $G$ is reductive, this last corollary is an easy consequence of IGUSA's Criterion [Igu73, Lemma 4].

We say that an affine $\mathbb{k}$-scheme $Z=\operatorname{Spec} R$ is a cone with apex $z_{0}$, if $R=$ $\bigoplus_{i>0} R_{i}$ is a positively graded ring with $R_{0}=\mathbb{k}$ and $z_{0}$ is the homogeneous maximal ideal. Geometrically this means that $Z$ admits an action of the multiplicative group $\mathbb{G}_{m}:=\mathbb{k}^{*}$ with a single closed orbit, namely the fixed point $z_{0}$. An affine variety $X$ is called a $G$-cone if $X$ is a cone and the $G$-action commutes with the $\mathbb{G}_{m}$-action. In particular, the apex $x_{0}$ is a fixed point for $G$. In this case $\left(X / / G, \pi\left(x_{0}\right)\right)$ is a cone, and the finite generation ideal $\mathfrak{f}_{X / / G}$ is homogeneous. 
Corollary 4.8. Let $\left(X, x_{0}\right)$ be a normal affine $G$-cone and $\left(Y, y_{0}\right)$ a normal affine cone. Assume that $\varphi: X \rightarrow Y$ is homogeneous, dominant and strongly separating. If $\pi\left(X_{0}\right) \in(X / / G)_{\text {alg }}$ and $\bar{\varphi}^{-1}\left(y_{0}\right)=\left\{\pi\left(x_{0}\right)\right\}$, then $\bar{\varphi}: X / / G \stackrel{\sim}{\longrightarrow} Y$ is an isomorphism. In particular, $\mathcal{O}(X)^{G}$ is finitely generated.

Proof. The complement of $(X / / G)_{a l g}$ in $X / / G$ is a closed cone, hence empty, because it does not contain the apex. Thus $\mathcal{O}(X)^{G}$ is finitely generated. Since $\bar{\varphi}: X / / G \rightarrow Y$ is homogeneous and $\bar{\varphi}^{-1}\left(y_{0}\right)=\left\{\pi\left(x_{0}\right)\right\}$ it follows that $\bar{\varphi}$ is finite (see e.g. [ZS60, Ch. VII, page 198, Lemma]). By Theorem 4.3(f) it is also an open immersion, hence an isomorphism.

Note that the special case of Corollary 4.8 for a representation of a reductive group $G$ is contained in [DK02, Proposition 2.3.12].

Proof of Theorem 4.3. The proof needs some preparation.

Lemma 4.9. Let $W$ be an irreducible affine variety, $R \subseteq \mathcal{O}(W)$ a $\mathbb{k}$-subalgebra and $\psi: W \rightarrow Z:=\operatorname{Spec} R$ the induced morphism. Then there is an $f \in \mathfrak{f}_{R}$ and a finite surjective morphism $\rho: W_{f} \rightarrow Z_{f} \times \mathbb{k}^{m}$, where $m:=\operatorname{dim} W-\operatorname{tdeg}_{\mathbb{k}} Q(R)$, such that $\left.\psi\right|_{W_{f}}=\operatorname{pr}_{Z_{f}} \circ \rho$ :

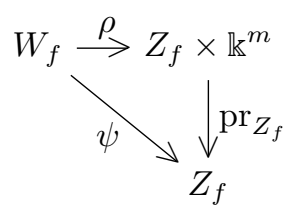

In particular, there is a subset $U \subseteq \psi(W)$ which is open, algebraic and dense in $Z$.

Proof. By first inverting some $f \in \mathfrak{f}_{R}$ we can assume that $R$ is finitely generated. In this case the result is well known, cf. [Bou98, Chap. V.3.1, Corollary 1].

The next two results can be found in [Win03, Lemma 1, 2, and 6]. The first is due to Nagata [Nag65].

Lemma 4.10. The invariant ring $R:=\mathcal{O}(X)^{G}$ is a KRULL-ring, i.e., it satisfies the following conditions:

(a) $R=\bigcap_{\mathfrak{p} \in \mathcal{P}} R_{\mathfrak{p}}$ where $\mathcal{P}$ is the set of the primes of $R$ of height 1 ;

(b) $R_{\mathfrak{p}}$ is a discrete valuation ring for all $\mathfrak{p} \in \mathcal{P}$;

(c) For any nonzero $r \in R$ the set $\{\mathfrak{p} \in \mathcal{P} \mid \mathfrak{p} \ni r\}$ is finite.

(A reference for KRULL-rings is [Mat89, §12].)

Lemma 4.11. Let $S \subset X / / G$ be an irreducible closed subscheme of codimension 1, and put $H:=\pi^{-1}(S) \subset X$. Then $S=\overline{\pi(H)}$.

Lemma 4.12. For any $r \in \mathcal{O}(X)^{G}$ we have $\mathcal{V}_{X / / G}(r)=\overline{\pi\left(\mathcal{V}_{X}(r)\right)}$.

Proof. We can assume that $r$ is neither zero nor invertible. Then $r \mathcal{O}(X)^{G}=$ $\bigcap_{\text {finite }} \mathfrak{p}^{\left(n_{\mathfrak{p}}\right)}$ is a finite intersection of symbolic powers ([Mat89, $\S 12$, page 88, Corollary to Theorem 12.3]). Hence $\mathcal{V}_{X / / G}(r)=\bigcup_{\text {finite }} S_{i}$ where $S_{i}$ are irreducible closed subschemes of codimension 1 . Now the claim follows from the previous lemma.

We will also need the following result; the proof is easy and left to the reader. 
Lemma 4.13. Let $Y$ be an irreducible variety, $C \subset Y$ an irreducible closed subset of codimension $d$ and $U \subset Y$ a nonempty open set. Then there is a chain

$$
Y=C_{0} \supset C_{1} \supset \cdots \supset C_{d}=C
$$

of closed irreducible subsets such that

(i) $\operatorname{codim}_{Y} C_{j}=j$ for $j=0, \ldots, d$, and

(ii) $C_{j} \cap U \neq \emptyset$ for $j<d$.

Proof of Theorem 4.3. (a) Lemma 4.9 implies that there is an open set $U \subseteq A$ such that $\pi(U)$ is open, algebraic and dense in $\overline{\pi(A)}$, and that $\varphi(U)$ is open, algebraic and dense in $\overline{\varphi(A)}$. Now $\pi(U(\mathbb{k})) \rightarrow \varphi(U(\mathbb{k}))$ is bijective, since $\varphi$ is separating. As $\pi(U)$ and $\varphi(U)$ are algebraic, it follows that $\operatorname{dim} \overline{\pi(A)}=\operatorname{dim} \pi(U)=\operatorname{dim} \varphi(U)=$ $\operatorname{dim} \overline{\varphi(A)}$.

To get the equality for the codimensions, we choose a nonempty open subset $O \subseteq X$ such that $\pi(O)$ is open and algebraic in $X / / G$, and such that $U:=\varphi(O)$ is open in $Y$. From Lemma 4.13 there is a sequence $C_{0}=Y \supset C_{1} \supset \cdots \supset C_{d}=\overline{\varphi(A)}$ of closed irreducible subsets $C_{j}$ with $\operatorname{dim} C_{j}=\operatorname{dim} Y-j$ such that $C_{j} \cap U \neq \emptyset$ for $j<d$. Since $\bar{\varphi}: \pi(O) \rightarrow \varphi(O)$ is a bijective morphism of varieties, we see that, for $j<d, B_{j}:=\overline{\bar{\varphi}^{-1}\left(C_{j}\right) \cap \pi(O)}$ is irreducible of dimension $\operatorname{dim} Y-j$, and that $B_{j} \subset B_{j-1}$. It remains to see that $B_{d-1} \supseteq \pi(A)$, since this implies that $\operatorname{codim}_{X / / G} \overline{\pi(A)} \geq d=\operatorname{codim}_{Y} \overline{\varphi(A)}$. If not, using again Lemma 4.9 , we can find a subset $U \subset \pi(A)$ which is open and dense in $\overline{\pi(A)}$ and such that $U \cap B_{d-1}=\emptyset$. Then the image $\bar{\varphi}(U)$ is disjoint from $\bar{\varphi}\left(B_{d-1} \cap \pi(O)\right)$. Since $\overline{\bar{\varphi}\left(B_{d-1} \cap \pi(O)\right)}=C_{d-1}$, it follows that $\overline{\varphi(A)}=\overline{\bar{\varphi}(U)}$ is not contained in $C_{d-1}$, contradicting the assumption.

(b) The same argument as above shows that, for irreducible closed subsets $A, B \subset$ $X$ with $\overline{\pi(A)} \nsubseteq \overline{\pi(B)}$, we have $\overline{\varphi(A)} \nsubseteq \overline{\varphi(B)}$. It follows that the map $\pi(X) \rightarrow \varphi(X)$ is injective, hence bijective, and open, hence a homeomorphism.

(c) For $\mathfrak{p} \in X / / G$ we have $\mathfrak{p} \in C_{X}=(X / / G) \backslash \pi(X)$ if and only if $\overline{\pi\left(\mathcal{V}_{X}(\mathfrak{p})\right)} \varsubsetneqq \mathcal{V}(\mathfrak{p})$ where $\mathcal{V}(\mathfrak{p})$ denotes the zero set in $X / / G$. Assume now that $\operatorname{codim}_{X / / G} \overline{C_{X}}=1$. This means that $\overline{C_{X}}$ contains an irreducible closed subscheme $S$ of codimension 1 corresponding to a prime ideal $\mathfrak{p} \in C_{X}$ of height 1 . It follows that $\overline{\pi\left(\pi^{-1}(S)\right)} \varsubsetneqq S$, contradicting Lemma 4.11.

(e) Let $S \subset X / / G$ be an irreducible hypersurface and let $\mathfrak{p} \subset R:=\mathcal{O}(X)^{G}$ be the corresponding prime ideal of height 1. Then, by Lemma 4.11 and (b), $H:=\overline{\varphi(S)}$ is an irreducible hypersurface, and so the corresponding prime ideal $\mathfrak{p}^{\prime}:=\mathfrak{p} \cap \mathcal{O}(Y)$ has also height 1 . Moreover, $\mathcal{O}(Y)_{\mathfrak{p}^{\prime}} \subseteq R_{\mathfrak{p}} \varsubsetneqq Q(R)=Q\left(\mathcal{O}(X)^{G}\right)$, by construction. Since $\mathcal{O}(Y)_{\mathfrak{p}^{\prime}}$ is a discrete valuation ring this implies that $\mathcal{O}(Y)_{\mathfrak{p}^{\prime}}=R_{\mathfrak{p}}$. But every irreducible hypersurface $H \subset Y$ not contained in $\overline{C_{Y}}$ is of the form $\overline{\bar{\varphi}(S)}$, hence $\mathcal{O}\left(Y \backslash \overline{C_{Y}}\right)=\bigcap_{\mathfrak{p}^{\prime}=\mathfrak{p} \cap \mathcal{O}(Y)} \mathcal{O}(Y)_{\mathfrak{p}^{\prime}}=\bigcap_{\mathfrak{p}} R_{\mathfrak{p}}=R$, by Lemma 4.10.

(f) If $f \in I\left(\overline{C_{Y}}\right)$, then $Y_{f} \subset Y \backslash \overline{C_{Y}}$, and so $\mathcal{O}(Y)_{f} \supset \mathcal{O}\left(Y \backslash \overline{C_{Y}}\right)=\mathcal{O}(X)^{G}$ by (e). Thus $\bar{\varphi}$ induces an isomorphism $(X / / G)_{f} \simeq Y_{f}$, and so $(X / / G)_{f}$ is algebraic.

(g) By (b), $\bar{\varphi}: X / / G \backslash \overline{C_{X}} \rightarrow Y$ is injective and birational. Hence, for every open algebraic subset $U \subset X / / G$, the map $\bar{\varphi}: U \backslash \overline{C_{X}} \rightarrow Y$ is an open immersion, by ZARISKI's Main Theorem (see [Mum99, III.§9, page 209]). 
(d) By construction, $\overline{\varphi\left(\varphi^{-1}\left(\overline{C_{Y}}\right)\right)}$ does not contain a hypersurface, and neither does $\bar{\varphi}^{-1}\left(\overline{C_{Y}}\right)$ by Lemma 4.11 and (a). The claim now follows since $\bar{\varphi}^{-1}\left(\overline{C_{Y}}\right) \supset$ $X / / G \backslash(X / / G)_{a l g}$, as we have seen in the proof of (f).

\section{5. $\mathbb{G}_{a}$-Actions, Local Slices, and the Plinth Variety}

$\mathbb{G}_{a}$-bundles. From now on we assume that char $\mathbb{k}=0$. In this and the following sections we focus on $\mathbb{G}_{a}$-varieties, i.e., varieties with an action of the additive group $\mathbb{G}_{a} \simeq(\mathbb{k},+) . \mathrm{A} \mathbb{G}_{a}$-variety $X$ (not necessarily affine) is called a trivial $\mathbb{G}_{a}$-bundle if there is a $\mathbb{G}_{a}$-equivariant isomorphism $\mathbb{G}_{a} \times Y \stackrel{\sim}{\rightarrow} X$, or, equivalently, if there is a $\mathbb{G}_{a}$-equivariant morphism $X \rightarrow \mathbb{G}_{a}$. In this case, $Y$ can be identified with the orbit space $X / \mathbb{G}_{a}$, and the quotient morphism $\pi: X \rightarrow X / \mathbb{G}_{a}$ admits a section. If $X$ is affine, then $X / \mathbb{G}_{a}=\operatorname{Spec} \mathcal{O}(X)^{\mathbb{G}_{a}}$, and this is an algebraic variety.

The $\mathbb{G}_{a}$-variety $X$ is called a principal $\mathbb{G}_{a}$-bundle (for short, a $\mathbb{G}_{a}$-bundle) if there is a $\mathbb{G}_{a}$-invariant morphism $\pi: X \rightarrow Z$ and an open covering $Z=\bigcup_{i} U_{i}$ such that $p^{-1}\left(U_{i}\right) \rightarrow U_{i}$ is a trivial $\mathbb{G}_{a}$-bundle for all $i$. In this case, $Z$ can be identified with the orbit space $X / \mathbb{G}_{a}$ and the morphism $\pi$ has the usual universal properties. Again, if $X$ is affine, then $X / \mathbb{G}_{a}=\operatorname{Spec} \mathcal{O}(X)^{\mathbb{G}_{a}}$, and this is an algebraic variety.

Local slices. Now let $X$ be an affine $\mathbb{G}_{a}$-variety. The $\mathbb{G}_{a}$-action defines a locally nilpotent vector field $D \in \operatorname{Vec}(X):=\operatorname{Der}_{\mathbb{k}}(\mathcal{O}(X))$ which determines the $\mathbb{G}_{a}$-action. Its kernel coincides with the ring of invariants: $\operatorname{ker} D=\mathcal{O}(X)^{\mathbb{G}_{a}}$. If $s \in \mathcal{O}(X)^{\mathbb{G}_{a}}$ is a nonzero invariant and $s=D f$ for some $f \in \mathcal{O}(X)$, then $D\left(\frac{f}{s}\right)=1$ and thus the morphism

$$
\frac{f}{s}: X_{s} \rightarrow \mathbb{G}_{a}
$$

is $\mathbb{G}_{a}$-equivariant. Such morphisms are called local slices. It follows that the affine open set $X_{s}$ is a trivial $\mathbb{G}_{a}$-bundle, and $X_{s} / \mathbb{G}_{a}=\operatorname{Spec} \mathcal{O}\left(X_{s}\right)^{\mathbb{G}_{a}}$. In particular, $\mathcal{O}\left(X_{s}\right)^{\mathbb{G}_{a}}=\left(\mathcal{O}(X)^{\mathbb{G}_{a}}\right)_{s}$ is finitely generated and so $s$ belongs to the finite generation ideal: $s \in \mathfrak{f}_{X / / \mathbb{G}_{a}}$

Definition 5.1. Let $X$ be an affine $\mathbb{G}_{a}$-variety. The ideal $\mathfrak{p}_{X / / \mathbb{G}_{a}} \subset \mathcal{O}(X)^{\mathbb{G}_{a}}$ generated by all $s \in \mathcal{O}(X)^{\mathbb{G}_{a}}$ of the form $s=D f$ for some $f \in \mathcal{O}(X)$ is called the plinth ideal:

$$
\mathfrak{p}_{X / / \mathbb{G}_{a}}:=D(\mathcal{O}(X)) \cap \operatorname{ker} D \subseteq \mathcal{O}(X)^{\mathbb{G}_{a}} .
$$

The corresponding (reduced) closed subscheme $\mathcal{P}_{X / / G} \subset X / / G$ is called the plinth scheme of $X / / G$ whereas the zeros set $\mathcal{P}_{X}:=\mathcal{V}_{X}\left(\mathfrak{p}_{X / / \mathbb{G}_{a}}\right) \subset X$ is called the plinth variety of $X$. By definition, we have $\mathcal{P}_{X}=\pi^{-1}\left(\mathcal{P}_{X / / G}\right)$, and $\mathfrak{p}_{X / / \mathbb{G}_{a}} \subseteq \mathfrak{f}_{X / / \mathbb{G}_{a}}$.

The next result shows that outside the plinth variety the quotient morphism is a principal $\mathbb{G}_{a}$-bundle.

Proposition 5.2. We have $\pi\left(X \backslash \mathcal{P}_{X}\right)=X / / \mathbb{G}_{a} \backslash \mathcal{P}_{X / / \mathbb{G}_{a}}$ and this is an open algebraic variety of $X / / \mathbb{G}_{a}$. Moreover, the morphism $\pi: X \backslash \mathcal{P}_{X} \rightarrow X / / \mathbb{G}_{a} \backslash \mathcal{P}_{X / / \mathbb{G}_{a}}$ is a principal $\mathbb{G}_{a}$-bundle.

Proof. If $s=D f$ and $D s=0$, then $\pi\left(X_{s}\right)=\left(X / / \mathbb{G}_{a}\right)_{s}$, and this is an open subset of $X / / \mathbb{G}_{a}$ which is an affine variety. Since we can cover $X \backslash \mathcal{P}_{X}$ with finitely many $X_{s_{j}}$ we see that $\pi\left(X \backslash \mathcal{P}_{X}\right)=\bigcup_{s \in \mathfrak{p}_{X / / \mathbb{G}_{a}}}(X / / G)_{s}=X / / G \backslash \mathcal{P}_{X / / G}$ is also covered by finitely many open affine varieties $(X / / G)_{s_{j}}$, hence is a variety. It remains to see 
that $\pi$ separates the $\mathbb{G}_{a}$-orbits on $X \backslash \mathcal{P}_{X}$. This is clear for two orbits contained in the same $X_{s_{j}}$. If $O_{1} \subset X_{s_{j}}$ and $O_{2} \subset X_{s_{k}} \backslash X_{s_{j}}$, then the invariant $s_{j}$ vanishes on $\mathrm{O}_{2}$, but not on $O_{1}$.

Definition 5.3. Let $X$ be a $\mathbb{G}_{a}$-variety. Define $X_{b d} \subseteq X$ to be the union of all open $\mathbb{G}_{a}$-stable subsets $U$ which are trivial $\mathbb{G}_{a}$-bundles:

$$
X_{b d}:=\bigcup_{\substack{U \subseteq X \text { open } \\ U \text { a trivial }}} U .
$$

If $X$ is affine, it follows from Proposition 5.2 that $X \backslash \mathcal{P}_{X} \subseteq X_{b d}$. We will see later (Example 8.4) that the inclusion may be strict. However, this cannot happen if $X$ is factorial.

Proposition 5.4. Let $X$ be a factorial affine $\mathbb{G}_{a}$-variety. Then

$$
X_{b d}=X \backslash \mathcal{P}_{X}
$$

In particular, $\pi\left(X_{b d}\right) \subseteq X / / \mathbb{G}_{a}$ is an open subvariety, and $X_{b d} \rightarrow \pi\left(X_{b d}\right)$ is a principal $\mathbb{G}_{a}$-bundle.

Proof. In the definition of $X_{b d}$ we can assume that all $U_{i}$ are affine. Since $X$ is factorial, this implies that $U_{i}=X_{t_{i}}$ for a suitable invariant $t_{i}$. On the other hand, if $X_{t}$ is a trivial $\mathbb{G}_{a}$-bundle where $t \in \mathcal{O}(X)^{\mathbb{G}_{a}}$, then there is an $h \in \mathcal{O}\left(X_{t}\right)$ such that $D h=1$. Writing $h=f t^{-k}$ we see that $s:=t^{k}=D f$, and so $X_{s}=X_{t}$ is of the form above.

\section{The CASE OF A REPRESENTATION}

Representations and the null cone. Let $V$ be representation of $\mathbb{G}_{a}$ over a field $\mathbb{k}$ of characteristic zero. Then $V$ extends to a representation of $\mathrm{SL}_{2}:=\mathrm{SL}_{2}(\mathbb{k})$, where $\mathbb{G}_{a}$ is identified with the unipotent subgroup $U \subset \mathrm{SL}_{2}$ via $s \mapsto\left[\begin{array}{ll}1 & s \\ 0 & 1\end{array}\right]$, (see [Kra84, III.3.9]). It follows that the invariants $\mathcal{O}(V)^{\mathbb{G}_{a}}$ are finitely generated (WeITZEnBÖCK's Theorem, loc. cit.), and the multiplicative group $\mathbb{G}_{m}$ acts linearly on $V,(t, v) \mapsto t \cdot v$, via the identification $t \mapsto\left[{ }^{t}{ }_{t^{-1}}\right] \in T \subset \mathrm{SL}_{2}$. This defines a decomposition of $V$ into weight spaces:

$$
V=\bigoplus_{k} V_{k}, \quad V_{k}:=\left\{v \in V \mid t \cdot v=t^{k} v\right\} .
$$

Since the invariants are finitely generated, the quotient $V / / \mathbb{G}_{a}:=\operatorname{Spec} \mathcal{O}(X)^{\mathbb{G}_{a}}$ is an affine variety. As usual, the nullcone is defined by $\mathcal{N}=\mathcal{N}_{V}:=\pi^{-1}(\pi(0)) \subseteq V$. Recall that the WEYL-group $W \simeq \mathbb{Z} / 2 \mathbb{Z}$ of $\mathrm{SL}_{2}$ acts on the zero weight space $V_{0}=$ $V^{\mathbb{G}_{m}}$. The nontrivial element of $W$ is represented by the matrix $\sigma=\left[\begin{array}{cc}0 & -1 \\ 1 & 0\end{array}\right] \in \mathrm{SL}_{2}$.

Theorem 6.1. (a) $\mathcal{N}_{V}=V^{+}:=\bigoplus_{k>0} V_{k}$.

(b) $\mathcal{P}_{V}=V \backslash V_{b d}=V_{0} \oplus V^{+}$. In particular, $\mathcal{P}_{V}=\mathcal{N}_{V}$ if and only if the $\mathrm{SL}_{2}$-representation $V$ does not contain odd-dimensional irreducible representations. 
(c) The image $\pi\left(\mathcal{P}_{V}\right) \subset V / / \mathbb{G}_{a}$ is closed. The induced map $\left.\pi\right|_{\mathcal{P}_{V}}: \mathcal{P}_{V} \rightarrow \pi\left(\mathcal{P}_{V}\right)$ is given by the $\mathrm{SL}_{2}$-invariants and has a factorization

$$
\mathcal{P}_{V}=V^{+} \oplus V_{0} \stackrel{\mathrm{pr}}{\longrightarrow} V_{0} \stackrel{\pi_{0}}{\longrightarrow} V_{0} / W \stackrel{\bar{\pi}}{\longrightarrow} \pi\left(\mathcal{P}_{V}\right)
$$

where $\pi_{0}$ is the quotient by $W$ and $\bar{\pi}$ is finite and bijective.

Remark 6.2. Elmer and KoHLs [EK12] gave an explicit construction of separating sets for indecomposable representations, which were later extended to any representation by Dufresne, Elmer, and Sezer [DES14].

The proof of the theorem needs some preparation.

Invariants and covariants. Let $V$ be a representation of $\mathrm{SL}_{2}$. The graded coordinate ring $\mathcal{O}(V)=\bigoplus_{d>0} \mathcal{O}(V)_{d}$ is a locally finite and rational $\mathrm{SL}_{2}$-module. A homogeneous irreducible submodule $F \subset \mathcal{O}(V)_{d}$ is classically called a covariant of degree $d$ and weight $r$, where $r$ is the weight of the highest weight vector $f_{0}$ of $F$. This means that $f_{0}$ is a homogeneous $\mathbb{G}_{a}$-invariant and that $t \cdot f_{0}=t^{r} f_{0}$ for $t \in \mathbb{G}_{m}$. In particular, $\operatorname{dim} F=r+1$. Thus, we always have $r \geq 0$, and $r=0$ if and only if $f_{0}$ is an $\mathrm{SL}_{2}$-invariant. We will say that $f_{0}$ is a homogeneous $\mathbb{G}_{a}$-invariant of degree $d$ and weight $r$.

Clearly, the invariants $\mathcal{O}(V)^{\mathbb{G}_{a}}$ are linearly spanned by the homogeneous $\mathbb{G}_{a^{-}}$ invariants of degree $d$ and weight $r$ where $d, r \geq 0$. Moreover, the homogeneous $\mathbb{G}_{a}$-invariants of degree $d$ and weight $r>0$ linearly span the plinth ideal $\mathfrak{p}_{V}=$ ker $D \cap \operatorname{im} D$ where $D \in \operatorname{Vec}(V)$ is the locally nilpotent vector field corresponding to the $\mathbb{G}_{a}$-action (see Definition 5.1). This shows that the $\mathbb{G}_{a}$-invariants are generated by $\mathfrak{p}_{V}$ together with the $\mathrm{SL}_{2}$-invariants.

In the following, we denote by $V[n]$ the irreducible $\mathrm{SL}_{2}$-module of highest weight $n$, i.e., $\operatorname{dim} V[n]=n+1$. One can take $V[n]:=\mathbb{k}[x, y]_{n}$, the binary forms of degree $n$, with the standard linear action of $\mathrm{SL}_{2}$. It follows that the element $\sigma \in \mathrm{SL}_{2}$ representing the nontrivial element of the Weyl group acts trivially on $V[n]_{0}$ if $n$ is odd or $n \equiv 0(\bmod 4)$, and by $(-\mathrm{id})$ if $n \equiv 2(\bmod 4)$.

In the proof below we will need the following classical result from invariant theory of binary forms. Choose a basis of weight vectors of $V[n]$ such that $\mathcal{O}(V[n])=$ $\mathbb{k}\left[x_{0}, x_{1}, \ldots, x_{n}\right]$, where $x_{i}$ has weight $n-2 i$.

Lemma 6.3. As an $\mathrm{SL}_{2}$-module we have the CLEBSCH-GORDAN decomposition $\mathcal{O}(V[n])_{2} \simeq V[2 n] \oplus V[2 n-4] \oplus V[2 n-8] \oplus \cdots$. The corresponding quadratic $\mathbb{G}_{a}$-invariants $f_{k} \in V[2 n-4 k]^{\mathbb{G}_{a}}$ have weight $2 n-4 k$ and are of the form

$$
f_{k}=\alpha_{0} x_{0} x_{2 k}+\alpha_{1} x_{1} x_{2 k-1}+\cdots+\alpha_{k} x_{k}^{2}, k=0,1,2, \ldots,\lfloor n / 2\rfloor,
$$

where all coefficients $\alpha_{j}$ are nonzero.

Proof. For the binary forms $V[2 k]$ of even degree $2 k$ there is a unique quadratic $\mathrm{SL}_{2-}$ invariant which has the form $A=\gamma_{0} x_{0} x_{2 k}+\gamma_{1} x_{1} x_{2 k-1}+\cdots+\gamma_{k} x_{k}^{2} \in \mathbb{k}\left[x_{0}, \ldots, x_{k}\right]$ where all coefficients $\gamma_{i}$ are nonzero (see [Sch68, Satz 2.6]; the invariant $A$ is classically called "Apolare"). Now $\mathbb{k}\left[x_{0}, \ldots, x_{2 k}\right] \subset \mathbb{k}\left[x_{0}, \ldots, x_{n}\right]=\mathcal{O}(V[n])$ is a $\mathbb{G}_{a}$-stable subalgebra, hence $A$ is a quadratic $\mathbb{G}_{a}$-invariant in $\mathcal{O}(V[n])$ of weight $2 n-4 k$, and so $f_{k}$ is a multiple of $A$.

Proof of Theorem 6.1. (a) Denote by $\mathbb{k}^{2} \simeq V[1]$ the standard representation of $\mathrm{SL}_{2}$ and consider the closed embedding $V \hookrightarrow V \oplus \mathbb{k}^{2}$ given by $v \mapsto\left(v, e_{1}\right)$. Then we have 
the following diagram (see [Kra84, III.3.2]):

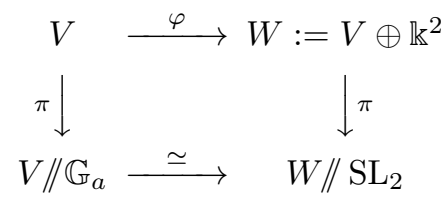

In particular, $\mathcal{N}_{V}=\varphi^{-1}\left(\mathcal{N}_{W}\right)=\mathcal{N}_{W} \cap V$. The HilBeRT-Criterion tells us that the elements $w=(v, a) \in \mathcal{N}_{W}$ are characterized by the condition that $0 \in \overline{\mathbb{G}_{m} g w}$ for a suitable $g \in \mathrm{SL}_{2}$ (see [Kra84, III.2.1]). This implies that $w=\left(v, e_{1}\right)$ belongs to $\mathcal{N}_{W}$ if and only if $0 \in \overline{\mathbb{G}_{m} v}$, i.e. if and only if $v \in V^{+}$.

(b) We first show that for every $v \in V \backslash\left(V^{+} \oplus V_{0}\right)$ there is a homogeneous $\mathbb{G}_{a}$-invariant $f$ of weight $>0$ such that $f(v) \neq 0$. For that we can assume that $V$ is irreducible, i.e., $V=V[n]$. We have $\mathcal{O}(V)=\mathbb{k}\left[x_{0}, x_{1}, \ldots, x_{n}\right]$, where $x_{i}$ has weight $n-2 i$. Thus $x_{i}$ vanishes on $V^{+}$if and only if $2 i \leq n$, and $x_{i}$ vanishes on $V^{+} \oplus V_{0}$ if and only if $2 i<n$.

Now let $v=\left(a_{0}, a_{1}, \ldots, a_{n}\right) \in V \backslash\left(V^{+} \oplus V_{0}\right)$, and let $a_{k}$ be the first nonzero coefficient. Then the quadratic $\mathbb{G}_{a}$-invariant $f_{k}$ from Lemma 6.3 above gives $f_{k}(v)=$ $\alpha_{k} a_{k}^{2} \neq 0$, and since $k<n / 2$ the $\mathbb{G}_{a}$-invariant $f_{k}$ has a positive weight.

It remains to show that every homogeneous $\mathbb{G}_{a}$-invariant $f$ of weight $>0$ vanishes on $V^{+} \oplus V_{0}$. But this is clear, because every monomial $m=x_{0}^{d_{0}} x_{1}^{d_{1}} \cdots x_{n}^{d_{n}}$ of positive weight must contain an $x_{i}$ of positive weight, i.e., with $2 i<n$. Hence $m$ vanishes on $V^{+} \oplus V_{0}$.

(c) The same argument shows that a homogeneous $\mathrm{SL}_{2}$-invariant restricted to $V^{+} \oplus V_{0}$ does not depend on $V^{+}$. This implies that the induced morphism

$$
\left.\pi\right|_{\mathcal{P}_{V}}: \mathcal{P}_{V} \rightarrow \pi\left(\mathcal{P}_{V}\right) \subseteq V / / \mathbb{G}_{a}
$$

is given by the $\mathrm{SL}_{2}$-invariants and has the following factorization

$$
\mathcal{P}_{V}=V^{+} \oplus V_{0} \stackrel{\mathrm{pr}}{\longrightarrow} V_{0} \stackrel{\left.\pi_{\mathrm{SL}_{2}}\right|_{V_{0}}}{\longrightarrow} \pi\left(\mathcal{P}_{V}\right)=\pi\left(V_{0}\right) \subset V / / \mathrm{SL}_{2}
$$

where $\pi_{\mathrm{SL}_{2}}: V \rightarrow V / / \mathrm{SL}_{2}$ is the quotient by $\mathrm{SL}_{2}$. The following lemma shows that $\left.\pi_{\mathrm{SL}_{2}}\right|_{V_{0}}$ induces a finite bijective morphism $V_{0} / W \rightarrow \pi\left(\mathcal{P}_{V}\right)$, as claimed.

The following general result was pointed out to us by the referee.

Lemma 6.4. Let $G$ be a connected reductive group with maximal torus $T$ and Weyl group $W$. For any affine $G$-variety $Z$ the natural map $Z^{T} / W \rightarrow Z / / G$ is finite and injective.

Proof. The finiteness follows from [Lun75, 2.1 Théorème]. Also, for any $z \in Z^{T}$, the orbit $G z \subset Z$ is closed, since the stabilizer contains a maximal torus, and $G z \cap X^{T}$ is a unique $W$-orbit, because all maximal tori in $G_{z}$ are conjugate.

\section{The Separating Variety}

Definitions. In section 4 , we discussed separating morphisms in the general context of a $G$-variety. We now introduce the separating variety $\mathcal{S}_{X}$ of a $G$-variety $X$, 
which measures how much the invariants separate the orbits (see [Kem03, Section 2]). Set

$\mathcal{S}_{X}:=\left\{(x, y) \in X \times X \mid f(x)=f(y)\right.$ for all $\left.f \in \mathcal{O}(X)^{G}\right\}=\bigcup_{z \in X / / G} \pi^{-1}(z) \times \pi^{-1}(z)$,

where $\pi: X \rightarrow X / / G$ is the quotient morphism. More schematically, the separating variety of $X$ is the reduced fiber product $\left(X \times_{X / / G} X\right)_{\text {red }}$. If $Y \subseteq X$ is a $G$-stable subvariety, we write $\mathcal{S}_{X, Y}:=\mathcal{S}_{X} \cap(Y \times Y)$.

The separating variety $\mathcal{S}_{X}$ contains the closure of the graph

$$
\Gamma_{X}:=\{(g x, x) \mid g \in G, x \in X\}=\bigcup_{x \in X} G x \times G x \subseteq X \times X .
$$

Note that $\Gamma_{X}=\mathcal{S}_{X}$ exactly when the quotient $\pi$ is almost geometric, i.e., when all nonempty fibers of $\pi$ are orbits. Also, if $\Gamma_{X}$ is closed, then all orbits are closed and have the same dimension. (The first statement is clear, and the second follows since $G x \times\{x\}=p_{2}^{-1}(x)$ where $p_{2}: \Gamma_{X} \rightarrow X$ is the second projection.)

More generally, we have the following result, which is a first step to determine the closure $\overline{\Gamma_{X}}$ and to decide whether $\overline{\Gamma_{X}}=\mathcal{S}_{X}$. For simplicity, we assume that $G$ is connected which implies that $\overline{\Gamma_{X}}$ is irreducible.

Proposition 7.1. Let $G$ be connected and $X$ a normal affine $G$-variety. Assume that there is a dense open set $U \subseteq X / / G$ such that $\varphi^{-1}(u)$ is nonempty and contains a dense orbit for all closed points $u \in U$. Set $X^{\prime}:=\pi^{-1}(U) \subseteq X$ and $P:=X \backslash X^{\prime}$.

(a) $\mathcal{S}_{X, P}$ is closed and $\mathcal{S}_{X}=\overline{\Gamma_{X}} \cup \mathcal{S}_{X, P}$. In particular, $\overline{\Gamma_{X}}$ is an irreducible component of $\mathcal{S}_{X}$.

(b) If $\pi^{-1}(u)$ is a single orbit for every closed point $u \in U$, then

$$
\mathcal{S}_{X}=\Gamma_{X^{\prime}} \cup \mathcal{S}_{X, P}=\Gamma_{X} \cup \mathcal{S}_{X, P}=\overline{\Gamma_{X}} \cup \mathcal{S}_{X, P} .
$$

(c) Assume in addition that $X^{\prime}$ is smooth, that the $G$-action on $X^{\prime}$ is free, and that $\operatorname{codim}_{X} P>1$. Then either $\Gamma_{X}$ is closed, or $\overline{\Gamma_{X}} \backslash \Gamma_{X^{\prime}}$ has codimension 1 in $\overline{\Gamma_{X}}$.

Proof. (a) If $X / / G$ is the disjoint union $O \cup A$, where $U$ is open and $A$ closed, then $\mathcal{S}_{X}=\mathcal{S}_{X, \pi^{-1}(O)} \cup \mathcal{S}_{X, \pi^{-1}(A)}$ where $\mathcal{S}_{X, \pi^{-1}(O)}$ is open, $\mathcal{S}_{X, \pi^{-1}(A)}$ is closed, and the union is disjoint. Take $(x, y) \in \mathcal{S}_{X, X^{\prime}}$. Then $\pi(x)=\pi(y)=: u \in U$. By assumption, the fiber $\pi^{-1}(u)$ contains a dense orbit, say $\overline{G z}=\pi^{-1}(u)$. Hence,

$$
(x, y) \in \pi^{-1}(u) \times \pi^{-1}(u)=\overline{G z} \times \overline{G z}=\overline{G z \times G z} \subseteq \overline{\Gamma_{X^{\prime}}}=\overline{\Gamma_{X}} .
$$

It follows that $\mathcal{S}_{X}=\mathcal{S}_{X, X^{\prime}} \cup \mathcal{S}_{X, P}=\overline{\Gamma_{X}} \cup \mathcal{S}_{X, P}$.

(b) Since the fibers over $U$ are orbits, we get $\mathcal{S}_{X, X^{\prime}}=\Gamma_{X^{\prime}}=\Gamma_{X} \cap\left(X^{\prime} \times X^{\prime}\right)$, and so

$$
\mathcal{S}_{X}=\mathcal{S}_{X, X^{\prime}} \cup \mathcal{S}_{X, P}=\Gamma_{X^{\prime}} \cup \mathcal{S}_{X, P}
$$

The claim follows.

(c) Consider the morphism $\mu: G \times X \rightarrow X \times X,(g, x) \mapsto(g x, x)$, whose image is $\Gamma_{X}$. By assumption, it induces an isomorphism $\mu_{0}: G \times X^{\prime} \stackrel{\sim}{\rightarrow} \Gamma_{X^{\prime}}$, and thus, a birational morphism $\tilde{\mu}: G \times X \rightarrow \tilde{\Gamma}$, where $\tilde{\Gamma} \rightarrow \overline{\Gamma_{X}}$ is the normalization. If $\operatorname{codim}_{\overline{\Gamma_{X}}} \overline{\Gamma_{X}} \backslash \Gamma_{X^{\prime}}>1$, then by IGUSA's criterion [Igu73], $\tilde{\mu}$ is an isomorphism, and so $\Gamma_{X}$ is closed. 
Remark 7.2. The first statement of the proposition above has the following converse: If $\overline{\Gamma_{X}}$ is an irreducible component of $\mathcal{S}_{X}$, then the general fiber of $\pi: X \rightarrow X / / G$ contains a dense orbit.

In order to see this, we can replace $X / / G$ be a dense open set and thus assume that $X / / G$ is affine algebraic, that $\pi: X \rightarrow X / / G$ is flat, and that the fibers are irreducible of dimension $n$. Then every irreducible component of $\mathcal{S}_{X}=X \times_{X / / G} X$ has dimension $2 \operatorname{dim} X-\operatorname{dim} X / / G=\operatorname{dim} X+n$ (see [Har77, Cor. 9.6 in Chap. III]). On the other hand, $\operatorname{dim} \overline{\Gamma_{X}}=\operatorname{dim} X+d$ where $d:=\max \{\operatorname{dim} G x \mid x \in X\}$. Hence $n=d$ and so the general fiber contains a dense orbit.

The case of $\mathbb{G}_{a}$-varieties. If $X$ is a $\mathbb{G}_{a}$-variety, then, by Proposition 5.2 , the quotient $\pi: X \backslash \mathcal{P}_{X} \rightarrow \pi\left(X \backslash \mathcal{P}_{X}\right)$ is a $\mathbb{G}_{a}$-bundle. This implies the following corollary.

Corollary 7.3. If $X$ is a normal affine $\mathbb{G}_{a}$-variety, then

$$
\mathcal{S}_{X}=\Gamma_{X \backslash \mathcal{P}_{X}} \cup \mathcal{S}_{X, \mathcal{P}_{X}}=\Gamma_{X} \cup \mathcal{S}_{X, \mathcal{P}_{X}}=\overline{\Gamma_{X}} \cup \mathcal{S}_{X, \mathcal{P}_{X}},
$$

and $\overline{\Gamma_{X}}$ is an irreducible component of $\mathcal{S}_{X}$.

In the remaining part of this section, we determine the irreducible components of $\mathcal{S}_{V}$ for a representation $V$ of $\mathbb{G}_{a}$ (cf. [DK13], where this is done for indecomposable representations). We have seen in Theorem 6.1(c) that the image $\pi\left(\mathcal{P}_{V}\right) \subset V / / \mathbb{G}_{a}$ is closed and the induced morphism $\left.\pi\right|_{\mathcal{P}_{V}}: \mathcal{P}_{V} \rightarrow \pi\left(\mathcal{P}_{V}\right)$ has a factorization

$$
\mathcal{P}_{V}=V^{+} \oplus V_{0} \stackrel{\mathrm{pr}}{\longrightarrow} V_{0} \stackrel{\pi_{0}}{\longrightarrow} V_{0} / W \stackrel{\bar{\pi}}{\longrightarrow} \pi\left(\mathcal{P}_{V}\right),
$$

where $\pi_{0}$ is the linear projection onto $W$ and $\bar{\pi}$ is finite and bijective. If $v \in \mathcal{P}_{V}=$ $V_{0} \oplus V^{+}$, we denote by $v_{0}$ the component of $v$ in $V_{0}$. Define the following closed subsets of $\mathcal{S}_{\mathcal{P}_{V}}$ :

$$
C:=\left\{\left(v, v^{\prime}\right) \in \mathcal{P}_{V} \times \mathcal{P}_{V} \mid v_{0}^{\prime}=v_{0}\right\}, \quad C_{\sigma}:=\left\{\left(v, v^{\prime}\right) \in \mathcal{P}_{V} \times \mathcal{P}_{V} \mid v_{0}^{\prime}=\sigma\left(v_{0}\right)\right\} .
$$

Both are irreducible and isomorphic to $V_{0} \times\left(V^{+} \times V^{+}\right)$. Now the factorization $(*)$ implies the following result.

Lemma 7.4. (a) If $\sigma$ acts trivially on $V_{0}$, then $\mathcal{S}_{\mathcal{P}_{V}}=C=C_{\sigma}$ is irreducible.

(b) If $\sigma$ acts nontrivially on $V_{0}$, then $\mathcal{S}_{\mathcal{P}_{V}}=C \cup C_{\sigma}$ has two irreducible components.

In particular, $\mathcal{S}_{\mathcal{P}_{V}}$ is equidimensional of dimension $\operatorname{dim} V$.

Now we can formulate our main result about the separating variety $\mathcal{S}_{V}$.

Theorem 7.5. We have $\mathcal{S}_{V}=\overline{\Gamma_{V}}$ if and only if the Weyl group acts trivially on $V_{0}$, or if $V=V[2] \oplus \mathbb{k}^{m}$. Otherwise, $\mathcal{S}_{V}$ has two irreducible components:

$$
\mathcal{S}_{V}=\overline{\Gamma_{V}} \cup C,
$$

where $\operatorname{dim} \overline{\Gamma_{V}}=\operatorname{dim} V+1$ and $\operatorname{dim} C=\operatorname{dim} V$.

Proof. We can assume that $V^{\mathrm{SL}_{2}}=(0)$. In fact, if $V=W \oplus \mathbb{k}^{m}$, then $\Gamma_{V}=\Gamma_{W} \times \mathbb{k}^{m}$ and $\mathcal{S}_{V}=\mathcal{S}_{W} \times \mathbb{k}^{m}$. It is easy to see that for $V=V[2]$ we have $\mathcal{S}_{V}=\overline{\Gamma_{V}}$. In all other cases, we have $\operatorname{dim} V^{+} \geq 2$ which implies that the component $C$ is not contained in $\Gamma_{V}$. On the other hand, $\overline{\Gamma_{V}}=\Gamma_{V} \cup C_{\sigma}$ by Lemma 7.6 below, and the claim follows from Lemma 7.4.

Lemma 7.6. We have $\overline{\Gamma_{V}}=\Gamma_{V} \cup C_{\sigma}$. 
The proof needs some preparation. If $X$ is a variety and $R$ a $\mathbb{k}$-algebra, we define the $R$-valued points by $X(R):=\operatorname{Mor}(\operatorname{Spec} R, X)$. Denote by $\mathbb{k} \llbracket t \rrbracket$ the power series ring and by $\mathbb{k}((t))$ its field of fractions. We have a canonical inclusion $X(\mathbb{k} \llbracket t \rrbracket) \subset$ $X(\mathbb{k}((t)))$ and a canonical map $X(\mathbb{k} \llbracket t \rrbracket) \rightarrow X(\mathbb{k})=X$ which will be denoted by $x=x(t) \mapsto x(0)=\left.x\right|_{t=0}$. We will constantly use the following known fact. For completeness we include a short proof.

Lemma 7.7. If $\varphi: X \rightarrow Y$ is a morphism and $y \in \overline{\varphi(X)}$, then there is an $x=$ $x(t) \in X(\mathbb{k}((t)))$ such that $\varphi(x) \in Y(\mathbb{k} \llbracket t \rrbracket)$ and $\left.\varphi(x)\right|_{t=0}=y$. Moreover, if $y \notin \varphi(X)$, then $x \notin X(\mathbb{k} \llbracket t \rrbracket)$.

Proof. We first claim that there is an irreducible curve $D \subset Y$ such that $y \in D$ and $D \cap \varphi(X)$ is open and dense in $D$. This is obvious if $Y=\mathbb{k}^{n}$. In general, we can assume that $Y$ is normal and $\operatorname{dim} Y>1$. Then we choose a finite surjective morphism $\psi: Y \rightarrow \mathbb{k}^{n}$ and use the Going-down property of $\psi$ to show that there is an irreducible hypersurface in $H \subset Y$ which contains $y$ and meets $\varphi(X)$ in a dense set. Now the claim follows by induction. (A stronger result can be found in [Mum08, Lemma on page 56]: Any two points of an irreducible variety can be connected by an irreducible curve.)

As a consequence we see that there is a smooth curve $C$, a point $c \in C$ and a morphism $\rho: C \backslash\{c\} \rightarrow X$ such that $\varphi \circ \rho: C \backslash\{c\} \rightarrow Y$ extends to a morphism $\tilde{\rho}: C \rightarrow Y$ with $\tilde{\rho}(c)=y:$

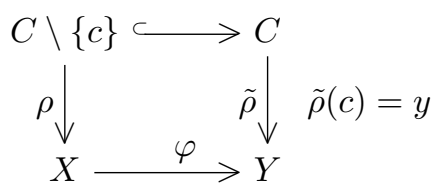

The completion $\hat{\mathcal{O}}_{C, c}$ is isomorphic to $\mathbb{k} \llbracket t \rrbracket$ and the corresponding point $p=p(t) \in$ $C(\mathbb{k} \llbracket t \rrbracket)$ has the property $p(0)=c$. Clearly, $p \in(C \backslash\{c\})(\mathbb{k}((t)))$, and so $x:=\rho(p) \in$ $X(\mathbb{k}((t)))$ has the required property: $\varphi(x)=\tilde{\rho}(p) \in Y(\mathbb{k} \llbracket t \rrbracket)$ and $\left.\varphi(x)\right|_{t=0}=\tilde{\rho}(c)=y$. If $x \in X(\mathbb{k} \llbracket t \rrbracket)$, then $y=\left.\varphi(x)\right|_{t=0}=\varphi(x(0)) \in \varphi(X)$, proving the second claim.

Proof of Lemma 7.6. We know from Proposition 7.1 that $E:=\overline{\Gamma_{V}} \cap \mathcal{S}_{\mathcal{P}_{V}}=\overline{\Gamma_{V}} \backslash \Gamma_{V_{b d}}$ has codimension 1 in $\overline{\Gamma_{V}}$, hence $E$ is either $C, C_{\sigma}$, or $C \cup C_{\sigma}$ by Lemma 7.4.

We now show that $\overline{\Gamma_{V}} \backslash \Gamma_{V} \subseteq C_{\sigma}$, which implies that $\overline{\Gamma_{V}}=\Gamma_{V} \cup C_{\sigma}$, hence the claim. Let $\left(v^{\prime}, v^{\prime \prime}\right) \in \overline{\Gamma_{V}} \backslash \Gamma_{V} \subseteq \mathcal{S}_{\mathcal{P}_{V}}$. Since $\Gamma_{V}$ is the image of the morphism $\mu: \mathbb{G}_{a} \times V \rightarrow V \times V,(s, v) \mapsto(s v, v)$, there are element $s(t) \in \mathbb{G}_{a}(\mathbb{k}((t))) \backslash \mathbb{G}_{a}(\mathbb{k} \llbracket t \rrbracket)$ and $v(t) \in V(\mathbb{k} \llbracket t \rrbracket)$ such that the following holds:

(a) $v(0)=v^{\prime \prime}$

(b) $s(t) v(t) \in V(\mathbb{k} \llbracket t \rrbracket)$ and $\left.(s(t) v(t))\right|_{t=0}=v^{\prime}$.

If $v_{0}^{\prime \prime} \in\left(V_{0}\right)^{\sigma}$, then $v_{0}^{\prime}=v_{0}^{\prime \prime}=\sigma v_{0}^{\prime \prime}$, and so $\left(v^{\prime}, v^{\prime \prime}\right) \in C_{\sigma}$. Thus we can assume that $v_{0}^{\prime \prime}$ is not fixed by $\sigma$, and we have to show that $v_{0}^{\prime}=\sigma v_{0}^{\prime \prime}=-v_{0}^{\prime \prime}$.

Now we use LunA's Slice Theorem in the point $v_{0}^{\prime \prime}$. Denote by $T \subset \mathrm{SL}_{2}$ the diagonal matrices identified with $\mathbb{G}_{m}$ as above, and by $U \subset \mathrm{SL}_{2}$ the upper triangular unipotent matrices, which we can identify with $\mathbb{G}_{a}$. There is a $T$-stable subspace $W \subset V$ containing $v_{0}^{\prime \prime}$ such that the morphism $\mu: \mathrm{SL}_{2} *_{T} W \rightarrow V$ given by $\mu([g, w]):=g w$ is étale in a $\mathrm{SL}_{2}$-saturated open neighborhood of $\left[e, v_{0}^{\prime \prime}\right]$ (see [Slo89]). Here the bundle $\mathrm{SL}_{2} *_{T} W$ is the quotient $\left(\mathrm{SL}_{2} \times W\right) / / T$ under the action $t(g, w):=\left(g t^{-1}, t w\right)$, and the quotient morphism $\mathrm{SL}_{2} \times W \rightarrow \mathrm{SL}_{2} *_{T} W$ is a principal $T$-bundle. Since principal $T$-bundles are locally trivial for the ZARISKI topology, 
this implies that we can lift the elements $v(t)$ and $s(t) v(t)$ to $\mathrm{SL}_{2} \times W$, i.e., there are elements $g(t) \in \mathrm{SL}_{2}(\mathbb{k} \llbracket t \rrbracket), w(t) \in W(\mathbb{k} \llbracket t \rrbracket)$ and $p(t) \in T(\mathbb{k}((t)))$ such that the following holds:

$\left(\mathrm{a}^{\prime}\right) g(t) w(t)=v(t)$, hence $g(0) w(0)=v^{\prime \prime}$;

$\left(\mathrm{b}^{\prime}\right) \tilde{g}(t):=s(t) g(t) p(t)^{-1} \in \mathrm{SL}_{2}(\mathbb{k} \llbracket t \rrbracket)$ and $\tilde{w}(t):=p(t) w(t) \in W(\mathbb{k} \llbracket t \rrbracket)$, hence $\tilde{g}(t) \tilde{w}(t)=s(t) v(t)$ and $\tilde{g}(0) \tilde{w}(0)=v^{\prime}$

Setting

$$
s(t)=\left[\begin{array}{cc}
1 & f(t) \\
0 & 1
\end{array}\right], \quad g(t)=\left[\begin{array}{cc}
a(t) & b(t) \\
c(t) & d(t)
\end{array}\right], \quad p(t)=\left[\begin{array}{cc}
r(t) & 0 \\
0 & r(t)^{-1}
\end{array}\right],
$$

where $f(t) \in \mathbb{k}((t)) \backslash \mathbb{k} \llbracket t \rrbracket, a(t), b(t), c(t), d(t) \in \mathbb{k} \llbracket t \rrbracket$, and $r(t) \in \mathbb{k}((t))$, we get

$$
\tilde{g}(t)=s(t) g(t) p(t)^{-1}=\left[\begin{array}{cc}
r^{-1}(a+f c) & (b+d f) r \\
r^{-1} c & d r
\end{array}\right] .
$$

Obviously, $p(t) \notin T(\mathbb{k} \llbracket t \rrbracket)$, since $s(t) \notin U(\mathbb{k} \llbracket t \rrbracket)$. Thus either $r(t) \in t \mathbb{k} \llbracket t \rrbracket$ and $c(0)=$ 0 , or $r(t)^{-1} \in t \mathbb{k} \llbracket t \rrbracket$ and $d(0)=0$. In the first case we get

$$
g(0) \in\left\{\left[\begin{array}{ll}
* & * \\
0 & *
\end{array}\right] \in \mathrm{SL}_{2}\right\}=: B \text { and } \tilde{g}(0) \in\left\{\left[\begin{array}{cc}
* & * \\
* & 0
\end{array}\right] \in \mathrm{SL}_{2}\right\}=B \sigma
$$

and in the second

$$
g(0) \in B \sigma \text { and } \tilde{g}(0) \in B
$$

Moreover, since $\tilde{w}(t)=p(t) w(t)$, we get $\tilde{w}(0)_{0}=w(0)_{0}$. Also note that for any $b \in B$ and $u \in V_{0} \oplus V^{+}$we have $(b u)_{0}=u_{0}$.

Assume now that we are in case (1). Since $g(0) w(0)=v^{\prime \prime} \in V_{0} \oplus V^{+}$, we get $w(0) \in V_{0} \oplus V^{+}$, hence $w(0)_{0}=(g(0) w(0))_{0}=v_{0}^{\prime \prime}$. On the other hand, $\tilde{g}(0) \in B \sigma$ and $\tilde{g}(0) \tilde{w}(0)=v^{\prime} \in V_{0} \oplus V^{+}$, hence $\sigma \tilde{w}(0) \in V_{0} \oplus V^{+}$and $(\sigma \tilde{w}(0))_{0}=v_{0}^{\prime}$. Thus $v_{0}^{\prime}=\sigma \tilde{w}(0)_{0}=-\tilde{w}(0)_{0}=-w(0)_{0}=-v_{0}^{\prime \prime}$, i.e. $\left(v^{\prime}, v^{\prime \prime}\right) \in C_{\sigma}$, and the claim follows. Case (2) is similar.

\section{8. $\mathbb{G}_{a}$-ACTIONS ON $\mathrm{SL}_{2}$-VARIETIES}

In this section, we generalize some of the results obtained for representations of $\mathbb{G}_{a}$ to affine $\mathrm{SL}_{2}$-varieties. As in section 6 we identify $\mathbb{G}_{a}$ with the unipotent subgroup $U \subset \mathrm{SL}_{2}$ via $s \mapsto\left[\begin{array}{ll}1 & s \\ 0 & 1\end{array}\right]$, and $\mathbb{G}_{m}$ with the maximal torus $T \subset \mathrm{SL}_{2}$ via $t \mapsto\left[\begin{array}{cc}t & 0 \\ 0 & t^{-1}\end{array}\right]$. Thus every $\mathrm{SL}_{2}$-variety $X$ can be regarded as a $\mathbb{G}_{a}$-variety. These $\mathbb{G}_{a}$-varieties have some very special properties, e.g. the following classical result which was already used in the proof of Theorem 6.1 (see [Kra84, III.3.2]).

Lemma 8.1. Let $X$ be an affine $\mathrm{SL}_{2}$-variety and denote by $\mathbb{k}^{2}$ the standard representation of $\mathrm{SL}_{2}$. Then the closed $\mathbb{G}_{a}$-equivariant embedding $X \hookrightarrow X \times \mathbb{k}^{2}, x \mapsto$ $\left(x, e_{1}\right)$, induces an isomorphism $X / / \mathbb{G}_{a} \stackrel{\sim}{\rightarrow}\left(X \times \mathbb{k}^{2}\right) / / \mathrm{SL}_{2}$. In particular, the $\mathbb{G}_{a^{-}}$ invariants $\mathcal{O}(X)^{\mathbb{G}_{a}}$ are finitely generated.

An immediate consequence is that for every closed embedding $X \hookrightarrow Y$ of affine $\mathrm{SL}_{2}$-varieties the induced map $X / / \mathbb{G}_{a} \rightarrow Y / / \mathbb{G}_{a}$ is also a closed embedding.

Proposition 8.2. Let $V$ be a representation of $\mathrm{SL}_{2}$ and $X \subset V$ a closed $\mathrm{SL}_{2}$-stable subset. 
(a) $\mathcal{S}_{X}=\mathcal{S}_{V} \cap(X \times X)$.

(b) For any $v \in\left(V_{0} \oplus V^{+}\right) \cap X$ we have $v_{0} \in X$.

(c) $\mathcal{P}_{X}=\mathcal{P}_{V} \cap X$. More precisely, the image of the plinth ideal $\mathfrak{p}_{V}$ under the restriction map is the plinth ideal $\mathfrak{p}_{X / / \mathbb{G}_{a}}$.

(d) $\mathcal{S}_{X, \mathcal{P}_{X}}=\mathcal{S}_{V, \mathcal{P}_{V}} \cap(X \times X)$.

Proof. (a) The inclusion $\mathcal{S}_{X} \subseteq \mathcal{S}_{V} \cap(X \times X)$ is obvious. Take $\left(x, x^{\prime}\right) \in \mathcal{S}_{V} \cap(X \times X)$. We have $f(x)=f\left(x^{\prime}\right)$ for all $f \in \mathcal{O}(V)^{\mathbb{G}_{a}}$. Since every element in $\mathcal{O}(X)^{\mathbb{G}_{a}}$ is the restriction to $X$ of an element in $\mathcal{O}(V)^{\mathbb{G}_{a}}$, we get $h(x)=h\left(x^{\prime}\right)$ for all $h \in \mathcal{O}(X)^{\mathbb{G}_{a}}$, and so $\left(x, x^{\prime}\right) \in \mathcal{S}_{X}$.

(b) Note that $v_{0} \in \overline{\mathbb{G}_{m} v}$, and the claim follows, since $X$ is closed and $\mathrm{SL}_{2}$-stable.

(c) The restriction map $\mathcal{O}(V) \rightarrow \mathcal{O}(X)$ is $\mathrm{SL}_{2}$-equivariant and so the image of an irreducible $\mathrm{SL}_{2}$-subrepresentation $W \subset \mathcal{O}(V)$ is either (0) or isomorphic to $W$. Therefore, the generators of $\mathfrak{p}_{V / / \mathbb{G}_{a}}$ are mapped onto the generators of $\mathfrak{p}_{X / / \mathbb{G}_{a}}$.

(d) This is clear from what has been said so far.

Proposition 8.3. Let $V$ be a representation of $\mathrm{SL}_{2}$ and $X \subset V$ a closed $\mathrm{SL}_{2}$-stable subset. Set $X_{0}:=X^{\mathbb{G}_{m}}=X \cap V_{0}$. Then the following are equivalent:

(i) $\mathcal{S}_{X}=\overline{\Gamma_{X}}$;

(ii) $\overline{\Gamma_{X}}=\overline{\Gamma_{V}} \cap(X \times X)$ and $\left(x_{0}+V^{+}\right) \cap X=\mathbb{G}_{a} x_{0}$ for all $x_{0} \in X_{0} \backslash\left(X_{0}\right)^{\sigma}$.

Proof. Since $\Gamma_{X}=\Gamma_{V} \cap X$ and $\overline{\Gamma_{V}}=\Gamma_{V} \cup C_{\sigma}$ (Lemma 7.6), we get

$$
\overline{\Gamma_{X}} \subseteq \overline{\Gamma_{V}} \cap(X \times X)=\Gamma_{X} \cup\left(C_{\sigma} \cap(X \times X)\right) \subseteq \mathcal{S}_{X},
$$

and from $\mathcal{S}_{V}=\Gamma_{V} \cup C_{\sigma} \cup C$ we obtain

$$
\mathcal{S}_{X}=\mathcal{S}_{V} \cap(X \times X)=\Gamma_{X} \cup\left(C_{\sigma} \cap(X \times X)\right) \cup(C \cap(X \times X)) .
$$

Therefore, $\overline{\Gamma_{X}}=\mathcal{S}_{X}$ if and only if $\overline{\Gamma_{X}} \supseteq \overline{\Gamma_{V}} \cap(X \times X)$ and $\left(C \backslash C_{\sigma}\right) \cap(X \times X) \subset \Gamma_{X}$. But the latter condition is clearly equivalent to $\left(x_{0}+V^{+}\right) \cap X=\mathbb{G}_{a} x_{0}$ for all $x_{0} \in X_{0} \backslash\left(X_{0}\right)^{\sigma}$.

Example 8.4. Let $X:=\mathrm{SL}_{2} / T$ where $T$ is acting by right multiplication on $\mathrm{SL}_{2}$. This variety is the smooth 2-dimensional affine quadric $X=\mathcal{V}\left(x z-y^{2}+y\right) \subset \mathbb{k}^{3}$, and the quotient map is given by

$$
\pi_{\mathrm{SL}_{2}}: \mathrm{SL}_{2} \rightarrow X, \quad\left[\begin{array}{ll}
a & b \\
c & d
\end{array}\right] \mapsto(a b, a d, c d) .
$$

Clearly, $X$ is an $\mathrm{SL}_{2}$-variety where the action is induced by left multiplication on $\mathrm{SL}_{2}$, and thus a $\mathbb{G}_{a}$-variety. The quotient by $\mathbb{G}_{a}$ is $\mathbb{A}^{1}$, and the quotient map is given by

$$
\mathrm{SL}_{2} / T \ni\left[\begin{array}{ll}
a & b \\
c & d
\end{array}\right] T \mapsto c d, \text { i.e. } X \ni(x, y, z) \mapsto z .
$$

The plinth ideal is generated by $z$ and is reduced. The plinth variety $\mathcal{P}_{X}$ consists of the two orbits $O_{1}:=U T$ and $O_{2}:=U \sigma T$ where $\sigma:=\left[\begin{array}{cc}0 & -1 \\ 1 & 0\end{array}\right]$, and so $X_{a l g}=$ $X \backslash\left(O_{1} \cup O_{2}\right)$. Moreover, the induced morphisms $X \backslash O_{i} \rightarrow \mathbb{A}^{1}$ are both trivial $\mathbb{G}_{a}$-bundles, and so $X \backslash O_{i} \simeq \mathbb{A}^{2}$ for $i=1,2$. Thus $X_{b d}=X$, but $\pi: X \rightarrow \mathbb{A}^{1}$ is not a $\mathbb{G}_{a}$-bundle, because $\pi^{-1}(0)=O_{1} \cup O_{2}$. It follows that

$$
\Gamma_{X}=\bigcup_{O \text { orbit }} O \times O \text { is open in } \mathcal{S}_{X}=\Gamma_{X} \cup\left(O_{1} \times O_{2}\right) \cup\left(O_{2} \times O_{1}\right) .
$$

Since $\mathcal{S}_{X} \subset X \times X$ is the hypersurface defined by $f:=\pi \circ \mathrm{pr}_{1}-\pi \circ \mathrm{pr}_{2}$, the irreducible components of $\mathcal{S}_{X}$ have codimension 1 in $X \times X$, hence $\mathcal{S}_{X}=\overline{\Gamma_{X}}$. 
Example 8.5. Now let us look at $Y:=\mathrm{SL}_{2} / N$, where $N=T \cup \sigma T$ is the normalizer of $T$. Then $\sigma$ induces an automorphism of order 2 on $X=\mathrm{SL}_{2} / T$ commuting with the $\mathbb{G}_{a}$-action, and the automorphism - id on the quotient $X / / \mathbb{G}_{a}=\mathbb{A}^{1}$. Thus $Y=X /\langle\sigma\rangle$ and $Y / / \mathbb{G}_{a}=\mathbb{A}^{1} /\{ \pm \mathrm{id}\} \simeq \mathbb{A}^{1}$. Since $\sigma\left(O_{1}\right)=O_{2}$ in the notation of Example 8.4 we see that the plinth variety $\mathcal{P}_{Y}=\pi^{-1}(0)$ is a single orbit, but the plinth ideal $\mathfrak{p}_{Y}$ is not prime. Therefore, $\pi: Y \rightarrow \mathbb{A}^{1}$ is a geometric quotient, but not a principal $\mathbb{G}_{a}$-bundle. In this case, $Y_{b d}=Y_{a l g}=X \backslash \mathcal{P}_{Y}$, and $\mathcal{S}_{Y}=\Gamma_{Y}$.

\section{Roberts' EXAMPLE}

In this section we discuss RoBERTs' counterexample to HILBERT's fourteenth problem [Rob90]. We assume that char $\mathbb{k}=0$ and define an action of the additive group $\mathbb{G}_{a}$ on $\mathbb{A}^{7}$ as follows:

$s \cdot\left(a_{1}, a_{2}, a_{3}, b_{1}, b_{2}, b_{3}, c\right):=\left(a_{1}, a_{2}, a_{3}, b_{1}+s a_{1}^{3}, b_{2}+s a_{2}^{3}, b_{3}+s a_{3}^{3}, c+s\left(a_{1} a_{2} a_{3}\right)^{2}\right)$.

It corresponds to the locally nilpotent vector field

$$
D:=x_{1}^{3} \frac{\partial}{\partial y_{1}}+x_{2}^{3} \frac{\partial}{\partial y_{2}}+x_{3}^{3} \frac{\partial}{\partial y_{3}}+\left(x_{1} x_{2} x_{3}\right)^{2} \frac{\partial}{\partial z},
$$

where we use the coordinates $\mathcal{O}\left(\mathbb{A}^{7}\right)=\mathbb{k}\left[x_{1}, x_{2}, x_{3}, y_{1}, y_{2}, y_{3}, z\right]$. Put $R:=\mathcal{O}\left(\mathbb{A}^{7}\right)^{\mathbb{G}_{a}}$ and let $\pi: \mathbb{A}^{7} \rightarrow \mathbb{A}^{7} / / \mathbb{G}_{a}:=\operatorname{Spec}(R)$ denote the quotient morphism.

The $x_{i}$ are invariants, and $D\left(y_{i}\right)=x_{i}^{3}$, hence $x_{i}^{3} \in \mathfrak{p}_{\mathbb{A}^{7} / / \mathbb{G}_{a}}$, and so

$$
\left(x_{1}, x_{2}, x_{3}\right) \subseteq \sqrt{\mathfrak{p}_{\mathbb{A}^{7} / / \mathbb{G}_{a}}} \subseteq \mathfrak{f}_{\mathbb{A}^{7} / / \mathbb{G}_{a}}
$$

(see Definition 5.1). It follows that $\mathbb{A}_{x_{i}}^{7} \rightarrow\left(\mathbb{A}^{7} / / \mathbb{G}_{a}\right)_{x_{i}}$ is a trivial $\mathbb{G}_{a}$-bundle for $i=1,2,3$. This allows to find the following additional invariants:

$$
\begin{gathered}
u_{12}:=x_{1}^{3} y_{2}-x_{2}^{3} y_{1}, u_{13}:=x_{1}^{3} y_{3}-x_{3}^{3} y_{1}, u_{23}:=x_{2}^{3} y_{3}-x_{3}^{3} y_{2}, \\
\beta_{11}:=x_{1} z-x_{2}^{2} x_{3}^{2} y_{1}, \beta_{21}:=x_{2} z-x_{1}^{2} x_{3}^{2} y_{2}, \beta_{31}:=x_{3} z-x_{1}^{2} x_{2}^{2} y_{3} .
\end{gathered}
$$

Define the following subalgebras of the ring of invariants $R$ :

$$
R_{0}:=\mathbb{k}\left[x_{1}, x_{2}, x_{3}, u_{12}, u_{13}, u_{23}\right] \subset R_{1}:=R_{0}\left[\beta_{11}, \beta_{21}, \beta_{31}\right] \subset R .
$$

We then have

$$
\left(R_{1}\right)_{x_{i}}=\mathcal{O}\left(\mathbb{A}^{7}\right)_{x_{i}}^{\mathbb{G}_{a}}=\mathcal{O}\left(\mathbb{A}_{x_{i}}^{7}\right)^{\mathbb{G}_{a}} \text { for } i=1,2,3 .
$$

Using a symbolic computation software like Singular [DGPS12], it is easy to see that $Y_{0}:=\operatorname{Spec} R_{0} \subset \mathbb{A}^{6}$ is the normal hypersurface defined by the equation $x_{1}^{3} u_{12}+x_{2}^{3} u_{13}+x_{3}^{3} u_{23}=0$, and that $Y:=\operatorname{Spec} R_{1} \subset \mathbb{A}^{9}$ has dimension 6 and its ideal $I(Y)$ is generated by the following 5 functions:

$$
\begin{gathered}
x_{1}^{2} u_{12}-x_{3} \beta_{21}+x_{2} \beta_{31}, x_{2}^{2} u_{13}-x_{1} \beta_{31}+x_{3} \beta_{11}, x_{3}^{2} u_{23}-x_{2} \beta_{11}+x_{1} \beta_{21}, \\
u_{12} u_{13} u_{23}\left(x_{1} x_{2} \beta_{31}+x_{2} x_{3} \beta_{11}+x_{3} x_{1} \beta_{21}\right)+u_{12} \beta_{11}^{3}+u_{13} \beta_{21}^{3}+u_{23} \beta_{31}^{3}, \\
x_{1} x_{2} x_{3} u_{12} u_{13} u_{23}+x_{1} u_{12} \beta_{11}^{2}+x_{2} u_{13} \beta_{21}^{2}+x_{3} u_{23} \beta_{31}^{2} .
\end{gathered}
$$

Remark 9.1. The given relations between the generators of $R_{0}$ and $R_{1}$ imply that the ideals $\left(x_{1}, x_{2}, x_{3}\right) R_{0} \subset R_{0}$ and $\left(x_{1}, x_{2}, x_{3}\right) R_{1} \subset R_{1}$ are prime, that

$$
\begin{gathered}
R_{0} /\left(x_{1}, x_{2}, x_{3}\right) \simeq \mathbb{k}\left[u_{12}, u_{13}, u_{23}\right] \text { and } \\
R_{1} /\left(x_{1}, x_{2}, x_{3}\right) \simeq \mathbb{k}\left[u_{12}, u_{13}, u_{23}, \beta_{11}, \beta_{12}, \beta_{13}\right] /\left(u_{12} \beta_{11}^{3}+u_{13} \beta_{21}^{3}+u_{23} \beta_{31}^{3}\right) .
\end{gathered}
$$


In particular, $\mathcal{V}_{Y_{0}}\left(x_{1}, x_{2}, x_{3}\right) \simeq \mathbb{A}^{3}$, and $\mathcal{V}_{Y}\left(x_{1}, x_{2}, x_{3}\right) \subset \mathbb{A}^{6}$ is a normal hypersurface of dimension 5 .

Lemma 9.2. The variety $Y$ is normal.

Proof. Again, using for example Singular [DGPS12], one verifies that the ideal $x_{1} R_{1}$ is radical. Let $f \in Q\left(R_{1}\right)$ be integral over $R_{1}$, that is, suppose $f$ satisfies an equation

$$
f^{d}=a_{1} f^{d-1}+a_{2} f^{d-2}+\cdots+a_{d}
$$

where $a_{i} \in R_{1}$. Since $\left(R_{1}\right)_{x_{1}}$ is normal, we have $x_{1}^{m} f \in R_{1}$ for some $m \geq 0$. We choose a minimal $m$ with this property. It follows from the equation above that $\left(x_{1}^{m} f\right)^{d} \in x_{1} R_{1}$, hence $x_{1}^{m} f \in x_{1} R_{1}$, and thus $f \in R_{1}$, because of the minimality of $m$.

The action of $\mathbb{G}_{a}$ on $\mathbb{A}^{7}$ commutes with the $\left(\mathbb{G}_{m}\right)^{3}$-action with weights

$$
(1,0,0),(0,1,0),(0,0,1),(3,0,0),(0,3,0),(0,0,3),(2,2,2),
$$

and so $\left(\mathbb{G}_{m}\right)^{3}$ also acts on $\mathbb{A}^{7} / / \mathbb{G}_{a}$. As the invariants $u_{i j}, \beta_{i j}$ are multihomogeneous, $\left(\mathbb{G}_{m}\right)^{3}$ also acts on $Y_{0}$ and $Y$.

The following two propositions collect the main properties of $\pi: \mathbb{A}^{7} \rightarrow \mathbb{A}^{7} / / \mathbb{G}_{a}$. Most statements follow from what we have done so far. The difficult part is the description of the finite generation ideal $\mathfrak{f}_{X / / \mathbb{G}_{a}}$.

Recall that $\mathcal{P}_{\mathbb{A}^{7}} \subset \mathbb{A}^{7}$ denotes the plinth variety, $\mathcal{P}_{\mathbb{A}^{7} / / \mathbb{G}_{a}} \subset \mathbb{A}^{7} / / \mathbb{G}_{a}$ the plinth scheme (Definition 5.1), and $\mathcal{S}_{\mathbb{A}^{7}} \subset \mathbb{A}^{7} \times \mathbb{A}^{7}$ the separating variety (section 7 ).

Proposition 9.3. $\quad$ (a) $\mathcal{P}_{\mathbb{A}^{7}}=\left(\mathbb{A}^{7}\right)^{\mathbb{G}_{a}}=\mathcal{V}_{\mathbb{A}^{7}}\left(x_{1}, x_{2}, x_{3}\right) \simeq \mathbb{A}^{4}$, and

$$
\mathbb{A}_{b d}^{7}=\mathbb{A}^{7} \backslash \mathcal{P}_{\mathbb{A}^{7}}=\mathbb{A}_{x_{1}}^{7} \cup \mathbb{A}_{x_{2}}^{7} \cup \mathbb{A}_{x_{3}}^{7} \text {. }
$$

(b) $\pi\left(\mathcal{P}_{\mathbb{A}^{7}}\right)=\pi\left(\left(\mathbb{A}^{7}\right)^{\mathbb{G}_{a}}\right)=\{\pi(0)\}$, and

$\pi\left(\mathbb{A}^{7}\right)=\left(\mathbb{A}^{7} / / \mathbb{G}_{a}\right)_{x_{1}} \cup\left(\mathbb{A}^{7} / / \mathbb{G}_{a}\right)_{x_{2}} \cup\left(\mathbb{A}^{7} / / \mathbb{G}_{a}\right)_{x_{3}} \cup\{\pi(0)\}=\pi\left(\mathbb{A}_{b d}^{7}\right) \cup\{\pi(0)\}$.

(c) The separating variety $\mathcal{S}_{\mathbb{A}^{7}}$ has two irreducible components:

$$
\mathcal{S}_{\mathbb{A}^{7}}=\overline{\Gamma_{\mathbb{A}^{7}}} \cup\left(\mathcal{P}_{\mathbb{A}^{7}} \times \mathcal{P}_{\mathbb{A}^{7}}\right),
$$

both of dimension 8.

(d) We have $\mathfrak{f}_{\mathbb{A}^{7} / / \mathbb{G}_{a}}=\sqrt{\left(x_{1}, x_{2}, x_{3}\right)}=\sqrt{\mathfrak{p}_{\mathbb{A}^{7} / / \mathbb{G}_{a}}}$, and so

$$
\left(\mathbb{A}^{7} / / \mathbb{G}_{a}\right)_{a l g}=\pi\left(\mathbb{A}_{b d}^{7}\right)=\pi\left(\mathbb{A}^{7}\right) \backslash\{\pi(0)\}=\mathbb{A}^{7} / / \mathbb{G}_{a} \backslash \mathcal{P}_{\mathbb{A}^{7} / / \mathbb{G}_{a}} .
$$

In particular, $\left(\mathbb{A}^{7} / / \mathbb{G}_{a}\right)_{\text {alg }}$ is algebraic.

(e) $\mathcal{P}_{\mathbb{A}^{7} / / \mathbb{G}_{a}}=\mathcal{V}_{\mathbb{A}^{7} / / \mathbb{G}_{a}}\left(x_{1}, x_{2}, x_{3}\right)$ is isomorphic to $\mathbb{A}^{3}$.

The inclusion $R_{1} \subset R$ defines an invariant morphism $\varphi: \mathbb{A}^{7} \rightarrow Y$ which factors through the quotient $\pi$ :

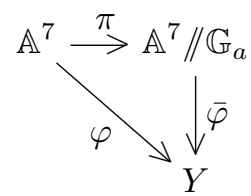

Proposition 9.4. $\quad$ (a) $\bar{\varphi}$ induces an isomorphism $\pi\left(\mathbb{A}_{b d}^{7}\right) \stackrel{\sim}{\rightarrow} \varphi\left(\mathbb{A}_{b d}^{7}\right)$.

(b) $Y$ is normal and $\varphi$ is a separating morphism.

(c) $C:=\mathcal{V}_{Y}\left(x_{1}, x_{2}, x_{3}\right) \subset Y$ is a hypersurface and $\mathcal{O}\left(\mathbb{A}^{7}\right)^{\mathbb{G}_{a}}=\mathcal{O}(Y \backslash C)$. 
(d) $\bar{\varphi}$ induces a closed immersion $\mathcal{P}_{\mathbb{A}^{7} / / \mathbb{G}_{a}} \hookrightarrow Y$ with image in $C$. In particular, $\bar{\varphi}: \mathbb{A}^{7} / / \mathbb{G}_{a} \rightarrow Y$ is injective.

A proof that $\varphi$ is a separating morphism and that (c) holds already appeared in [Duf13, Example 4.2].

Proof. Statement (a) holds since $\left(\mathbb{A}^{7} / / \mathbb{G}_{a}\right)_{x_{i}} \simeq Y_{x_{i}}$ by $(*)$ above. We have seen in Lemma 9.2 that $Y$ is normal, and the morphism $\bar{\varphi}$ is injective on $\pi\left(\mathbb{A}^{7}\right)$ since $\bar{\varphi}\left(\pi\left(x_{0}\right)\right)=\varphi\left(x_{0}\right) \in \mathcal{V}_{Y}\left(x_{1}, x_{2}, x_{3}\right)$, proving (b).

(c) follows from Theorem 4.3(e), because $Y \backslash \varphi\left(\mathbb{A}^{7}\right)=\mathcal{V}_{Y}\left(x_{1}, x_{2}, x_{3}\right) \backslash\{\varphi(0)\}$.

For $(\mathrm{d})$ we have to show that $R_{1} \stackrel{\bar{\varphi}}{\rightarrow} R \rightarrow R / \mathfrak{f}_{\mathbb{A}^{7} / / \mathbb{G}_{a}}=\mathcal{O}\left(\mathcal{P}_{\mathbb{A}^{7} / / \mathbb{G}_{a}}\right)$ is surjective and contains $x_{1}, x_{2}, x_{3}$ in its kernel. For this we use two results which will be proved below. By Proposition 9.3(d) we have $\mathfrak{f}_{\mathbb{A}^{7} / / G}=\sqrt{\left(x_{1}, x_{2}, x_{3}\right)}$. Hence, by Lemma 9.8, we get $R / \mathfrak{f}_{\mathbb{A}^{7} / / \mathbb{G}_{a}}=\mathbb{k}\left[\bar{u}_{12}, \bar{u}_{13}, \bar{u}_{23}\right]$, and so $R_{1} \rightarrow R / \mathfrak{f}_{\mathbb{A}^{7}}$ is surjective and contains $x_{1}, x_{2}, x_{3}$ in the kernel.

Proof of Proposition 9.3(a)-(c). We have $\pi^{-1}\left(\mathcal{V}_{\mathbb{A}^{7} / / \mathbb{G}_{a}}\left(x_{1}, x_{2}, x_{3}\right)\right)=\pi^{-1}(\pi(0))$, implying (a). As $\pi\left(\pi^{-1}\left(\mathcal{V}_{\mathbb{A}^{7} / / \mathbb{G}_{a}}\left(x_{1}, x_{2}, x_{3}\right)\right)\right)=\{\pi(0)\}$, (b) follows. Finally, (c) follows from (a) and Corollary 7.3.

The proofs of the remaining statements (d) and (e) need some preparation. They will be given at the end of the section.

To prove that $\mathcal{O}\left(\mathbb{A}^{7}\right)^{\mathbb{G}_{a}}$ is not finitely generated, RoBERTs showed in [Rob90, Lemma 3] that there exist invariants of the form

$$
x_{i} z^{n}+\text { terms of lower } z \text {-degree }
$$

for $i=1,2,3$ and $n \geq 0$. Later, KurodA proved (see [Kur04, Theorem 3.3]) that any set $S$ of such invariants, together with $u_{12}, u_{13}, u_{23}$, forms a SAGBI-basis for the lexicographic monomial ordering with $x_{1} \prec x_{2} \prec x_{3} \prec y_{1} \prec y_{2} \prec y_{3} \prec z$. We will improve this statement in Lemma 9.6 below.

Recall that if $R$ is a subalgebra of a polynomial ring, then for a given monomial ordering, a $S A G B I$-basis is a subset $S \subset R$ such that $\mathbb{k}[\operatorname{LT}(S)]=\mathbb{k}[\operatorname{LT}(R)]$ where LT $(S)$ denotes the set of leading terms of the polynomials in $S$ (see [RS90]). Such a basis always generates $R$. Note that for KuroDA's SAGBI-bases $S$ defined above we always have

$$
\operatorname{LT}(S)=\left\{x_{1}^{3} y_{2}, x_{1}^{3} y_{3}, x_{2}^{3} y_{3}, x_{j} z^{n} \mid j \in\{1,2,3\}, n \geq 0\right\} .
$$

Lemma 9.5. There exist invariants $\beta_{\text {in }}$ for $n \geq 0$ and $i=1,2,3$ which are multihomogeneous and of the form

$$
\begin{aligned}
& \beta_{\text {in }}= x_{i} z^{n}-n x_{j}^{2} x_{k}^{2} y_{i} z^{n-1}+ \\
&+\left(\begin{array}{c}
n \\
2
\end{array}\right)\left(x_{i}^{2} x_{j}^{4} x_{k} y_{i} y_{k}+x_{i}^{2} x_{j} x_{k}^{4} y_{i} y_{j}-x_{i}^{5} x_{j} x_{k} y_{j} y_{k}\right) z^{n-2}+ \\
&+ \text { terms of lower } z \text {-degree }
\end{aligned}
$$

Proof. By symmetry it suffices to look at the case $i=1$. We know from [Rob90, Lemma 3] that invariants $\beta_{1 n}$ with leading term $x_{1} z^{n}$ exist, and we can clearly assume that they are multihomogeneous of degree $(2 n+1,2 n, 2 n)$, hence

$$
\beta_{1 n}=x_{1} z^{n}+f_{1} z^{n-1}+f_{2} z^{n-2}+\text { terms of lower } z \text {-degree }
$$


where $f_{1}, f_{2} \in \mathbb{k}[x, y]:=\mathbb{k}\left[x_{1}, x_{2}, x_{3}, y_{1}, y_{2}, y_{3}\right], \operatorname{deg} f_{1}=(3,2,2)$ and $\operatorname{deg} f_{2}=$ $(5,4,4)$. From $D\left(\beta_{1 n}\right)=0$ we get the following differential equations

$$
\begin{aligned}
& D\left(f_{1}\right)=-n x_{1} D(z)=-n x_{1}^{3} x_{2}^{2} x_{3}^{2} \\
& D\left(f_{2}\right)=-(n-1) f_{1} D(z)=-(n-1) x_{1}^{2} x_{2}^{2} x_{3}^{2} f_{1},
\end{aligned}
$$

which have the special solutions

$$
h_{1}:=-n x_{2}^{2} x_{2}^{2} y_{1}, \text { and } \quad h_{2}:=\left(\begin{array}{l}
n \\
2
\end{array}\right)\left(x_{1}^{2} x_{2}^{4} x_{3} y_{1} y_{3}+x_{1}^{2} x_{2} x_{3}^{4} y_{1} y_{2}-x_{1}^{5} x_{2} x_{3} y_{2} y_{3}\right) .
$$

An easy calculation shows that $\operatorname{ker} D \cap \mathbb{k}[x, y]_{(3,2,2)}=\mathbb{k} x_{1}^{3} x_{2}^{2} x_{3}^{2}$, and so $f_{1}=h_{1}+$ $c x_{1}^{3} x_{2}^{2} x_{3}^{2}$ for some $c \in \mathbb{k}$. But then we may replace $\beta_{1 n}$ by $\beta_{1 n}-c x_{1}^{2} x_{2}^{2} x_{3}^{2} \beta_{1 n-1}$, which has the form $x_{1} z^{n}-n x_{j}^{2} x_{k}^{2} y_{i} z^{n-1}+$ terms of lower $z$-degree. Thus we can assume that $f_{1}=h_{1}$, and hence $f_{2}=h_{2}+c_{2}$, where $D\left(c_{2}\right)=0$. It is not difficult to see that

$$
\operatorname{ker} D \cap \mathbb{k}[x, y]_{(5,4,4)}=\mathbb{k} x_{1}^{5} x_{2}^{4} x_{3}^{4} \oplus \mathbb{k} x_{1}^{2} x_{2} x_{3}^{4} u_{12} \oplus \mathbb{k} x_{1}^{2} x_{2}^{4} x_{3} u_{13}
$$

Subtracting from $\beta_{1 n}$ a suitable linear combination of the invariants $x_{1}^{4} x_{2}^{4} x_{3}^{4} \beta_{1 n-2}$, $x_{1} x_{2} x_{3}^{4} u_{12} \beta_{1 n-2}$ and $x_{1} x_{2}^{4} x_{3} u_{13} \beta_{1 n-2}$, we can assume that $f_{2}=h_{2}$, and the claim follows.

Define $S_{N}:=\left\{u_{12}, u_{13}, u_{23}, \beta_{i n} \mid i=1,2,3\right.$, and $\left.0 \leq n \leq N\right\}$ and set $R_{N}:=$ $\mathbb{k}\left[S_{N}\right] \subset R$ for all $N \geq 0$, extending our definition of the subalgebras $R_{0}$ and $R_{1}$ above. One easily sees that $R_{0}$ is the ring formed by the invariants of $z$-degree 0 , that is, the invariants of the induced $\mathbb{G}_{a}$-action on the hyperplane $\mathcal{V}_{\mathbb{A}^{7}}(z) \subset \mathbb{A}^{7}$. The $R_{N}$ for $N \geq 1$ yield a family of separating morphisms $\varphi_{N}: \mathbb{A}^{7} \rightarrow Y_{N}:=\operatorname{Spec}\left(R_{N}\right)$, and, by KURODA's result mentioned above, we have $R=\bigcup_{N} R_{N}$.

The following lemma is crucial.

Lemma 9.6. For all $N \geq 0$ the subalgebra $\mathbb{k}\left[\operatorname{LT}\left(R_{N}\right)\right] \subset R$ is generated by $\operatorname{LT}\left(S_{N}\right)$. Equivalently, $S_{N}$ is a SAGBI-basis of $R_{N}$.

Proof. Put $b_{i n}:=\operatorname{LT}\left(\beta_{i n}\right)$ and $m_{i j}:=\operatorname{LT}\left(u_{i j}\right)$ :

$$
b_{\text {in }}=x_{i} z^{n}, \quad m_{12}=x_{1}^{3} y_{2}, \quad m_{13}=x_{1}^{3} y_{3}, \quad m_{23}=x_{2}^{3} y_{3} .
$$

(a) We first claim that the relations between these leading term are generated by

$$
b_{1 n} b_{1 m} b_{1 k} m_{23}-b_{2 n} b_{2 m} b_{2 k} m_{13}=0 \text { where } 0 \leq n \leq m \leq k \leq N \text {, and }
$$

$b_{i n} b_{j m}-b_{i n^{\prime}} b_{j m^{\prime}}=0$ where $0 \leq n \leq m \leq N, m+n=m^{\prime}+n^{\prime} \geq 1, i, j \in\{1,2,3\}$.

This is not difficult and we leave the details to the reader.

(b) It remains to show that, when we substitute the polynomials $\beta_{\text {in }}$ defined in Lemma 9.5 in the relations above, the leading term of the result belongs to $\mathbb{k}\left[\operatorname{LT}\left(S_{N}\right)\right]$, that is:

$$
\begin{gathered}
\operatorname{LT}\left(\beta_{1 n} \beta_{1 m} \beta_{1 k} u_{23}-\beta_{2 n} \beta_{2 m} \beta_{2 k} u_{13}\right) \in \mathbb{k}\left[\operatorname{LT}\left(S_{N}\right)\right], \text { and } \\
\operatorname{LT}\left(\beta_{i n} \beta_{j m}-\beta_{i n^{\prime}} \beta_{j m^{\prime}}\right) \in \mathbb{k}\left[\operatorname{LT}\left(S_{N}\right)\right] .
\end{gathered}
$$

(b1) A simple computation shows that $\beta_{1 n} \beta_{1 m} \beta_{1 k} u_{23}-\beta_{2 n} \beta_{2 m} \beta_{2 k} u_{13}$ has $z$ degree $n+m+k$ and leading term $-x_{1}^{3} x_{3}^{3} y_{2} z^{m+n+k}=-b_{3 n} b_{3 m} b_{3 k} m_{12}$, which is indeed in $\mathbb{k}\left[\operatorname{LT}\left(S_{N}\right)\right]$. 
(b2) A similar computation shows that $\beta_{1 n} \beta_{2 m}-\beta_{1 n^{\prime}} \beta_{2 m^{\prime}}$ has $z$-degree $n+m-1$ and leading term $\left(n-n^{\prime}\right) x_{1}^{3} x_{3}^{2} y_{2} z^{n+m-1}=\left(n-n^{\prime}\right) b_{3 n} b_{3 m-1} m_{12}$, which also belongs to $\mathbb{k}\left[\operatorname{LT}\left(S_{N}\right)\right]$.

(b3) It remains to consider $\beta_{1 n} \beta_{1 m}-\beta_{1 n^{\prime}} \beta_{1 m^{\prime}}$. For $m+n \leq 1$ this expression is 0 , and for $m+n \geq 2$ it has $z$-degree $m+n-2$ and leading term $(n m-$ $\left.n^{\prime} m^{\prime}\right) x_{1}^{6} x_{2} x_{3} y_{2} y_{3} z^{n+m-2}$ which is equal to $\left(m n-m^{\prime} n^{\prime}\right) m_{12} m_{13} b_{2 n-1} b_{3 m-1}$ if $n>0$ and to $\left(-m^{\prime} n^{\prime}\right) m_{12} m_{13} b_{20} b_{3 n-2}$ if $n=0$, with both belonging to $\mathbb{k}\left[\operatorname{LT}\left(S_{N}\right)\right]$.

For subalgebras $B_{1} \subset B_{2} \subset R$ the conductor is defined as usual by $\left[B_{1}: B_{2}\right]:=$ $\left\{b \in B_{2} \mid b B_{2} \subset B_{1}\right\}$.

Lemma 9.7. (a) If $f \in R$ and $\operatorname{deg}_{z} f \leq N$, then $f \in R_{N}$.

(b) $\left(x_{1}, x_{2}, x_{3}\right) R_{N+1} \subseteq R_{N}$.

(c) $\left[R_{N}: R_{N+1}\right] \cap R_{0}=\left(x_{1}, x_{2}, x_{3}\right) R_{0}$.

Proof. (a) This statement is clear for $N=0$. If $\operatorname{deg}_{z} f=N>0$, then LT $(f)$ is a monomial in $\operatorname{LT}(S)$ of $z$-degree $N$, and thus a monomial in $\operatorname{LT}\left(S_{N}\right)$. Now Lemma 9.6 implies that $\operatorname{LT}(f)=\operatorname{LT}(\tilde{f})$ for some $\tilde{f} \in R_{N}$. Thus $\operatorname{deg}_{z}(f-\tilde{f})<N$, and the claim follows by induction.

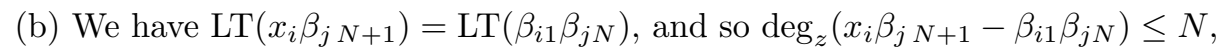
hence $\left(x_{i} \beta_{j+1}-\beta_{i 1} \beta_{j N}\right) \in R_{N}$ by (a), and thus $x_{i} \beta_{j+1} \in R_{N}$.

(c) Assume that $f R_{N+1} \subset R_{N}$ for some $f \in R_{0}$. Then $f \beta_{i N+1} \in R_{N}$ for all $i$, hence $\operatorname{LT}\left(f \beta_{i N+1}\right) \in \operatorname{LT}\left(R_{N}\right)$. Thus $\operatorname{LT}\left(f \beta_{i N+1}\right)=\operatorname{LT}(f) x_{i} z^{N+1}$ is a monomial in $\operatorname{LT}\left(S_{N}\right)$. It follows that this monomial contains at least two factors of the form $x_{j} z^{n}=\operatorname{LT}\left(\beta_{j n}\right)$. This implies that $\operatorname{LT}(f)$, as a monomial in $\operatorname{LT}\left(S_{0}\right)$, contains a factor $x_{j}$. Hence, $\operatorname{LT}(f)=x_{j} \operatorname{LT}(\tilde{f})$ for some $\tilde{f} \in R_{0}$, and so $f-x_{j} \tilde{f} \prec f$. Now the claim follows by induction since $x_{j} R_{N+1} \subset R_{N}$, by (b).

Lemma 9.8. If $f \in R$ is a multihomogeneous invariant whose multi-degree is not congruent to $(k, k, k)$ modulo 3 , then $f^{2} \in\left(x_{1}, x_{2}, x_{3}\right)$. In particular, $\beta_{j n}^{2} \in$ $\left(x_{1}, x_{2}, x_{3}\right) R$ for all $j \in\{1,2,3\}, n \geq 0$. Moreover, the radical $\mathfrak{p}:=\sqrt{\left(x_{1}, x_{2}, x_{3}\right) R}$ is generated by $\left\{\beta_{\text {in }}\right\}$ and $R / \mathfrak{p}=\mathbb{k}\left[\bar{u}_{12}, \bar{u}_{13}, \bar{u}_{23}\right]$ is a polynomial ring in 3 variables.

Proof. By induction, it suffices to show that $\operatorname{LT}\left(f^{2}\right)=\operatorname{LT}(h)$ where $h \in\left(x_{1}, x_{2}, x_{3}\right)$. But $\operatorname{LT}(f)$, as a monomial in $\operatorname{LT}(S)$, must contain a factor of the form $x_{i}$ or $x_{i} z$ since otherwise the multi-degree is congruent to $(k, k, k)$ modulo 3 . Hence, $\operatorname{LT}\left(f^{2}\right)$ contains a factor $x_{i}$, and so $\operatorname{LT}\left(f^{2}\right)=\operatorname{LT}\left(x_{i} p\right)$ for some $p \in R$.

Next we remark that $u_{i j} \notin \mathfrak{p}$, for all $i, j$. In fact, if $u_{i j}^{k} \in\left(x_{1}, x_{2}, x_{3}\right) R$, then $\operatorname{LT}\left(u_{i j}^{k}\right)=\operatorname{LT}\left(u_{i j}\right)^{k}$ is a monomial in $\operatorname{LT}\left(S_{0}\right)$ containing a factor $x_{j}$ which is impossible. Since $\beta_{\text {in }} \in \mathfrak{p}$ it follows that $R / \mathfrak{p}$ is generated by the (nonzero) images of $u_{12}, u_{13}, u_{23}$ which are algebraically independent, because their multi-degrees are linearly independent. Thus, $R / \mathfrak{p}$ is a polynomial ring in 3 variables, and $\mathfrak{p}$ is generated by $\left\{\beta_{\text {in }}\right\}$.

Proof of Proposition 9.3(d)-(e). For (d) we already know that $x_{1}, x_{2}, x_{3} \in \mathfrak{f}_{X / / \mathbb{G}_{a}}$, hence $\sqrt{\left(x_{1}, x_{2}, x_{3}\right)} \subseteq \mathfrak{f}_{X / / \mathbb{G}_{a}}$, and by Lemma 9.8 we have $\beta_{i n} \in \mathfrak{f}_{X / / \mathbb{G}_{a}}$ for all $i, n$. Now let $f \in \mathfrak{f}_{X / / \mathbb{G}_{a}}$. Since $R=R_{0}+\left(\beta_{i n}\right) R$ we can assume that $f \in R_{0}$. Since $R_{f}$ is finitely generated there is an $N>0$ such that $R_{f}=\left(R_{N}\right)_{f}$, and so $f^{k} \beta_{i N+1} \in R_{N}$ for some $k>0$ and all $i$. Hence $f^{k} \in\left(x_{1}, x_{2}, x_{3}\right) R$ by Lemma $9.7(\mathrm{c})$, and (d) follows.

Finally, (e) follows from the above and Lemma 9.8 . 


\section{REFERENCES}

[Bou98] Nicolas Bourbaki, Commutative algebra. Chapters 1-7, Elements of Mathematics (Berlin), Springer-Verlag, Berlin, 1998, Translated from the French, Reprint of the 1989 English translation.

[DGPS12] W. Decker, G.-M. Greuel, G. Pfister, and H. Schönemann, Singular 3-1-6 - A computer algebra system for polynomial computations, http://www.singular.uni-kl.de.

[DK02] Harm Derksen and Gregor Kemper, Computational invariant theory, Invariant Theory and Algebraic Transformation Groups, I, Springer-Verlag, Berlin, 2002, Encyclopaedia of Mathematical Sciences, 130.

[DK08] _ Computing invariants of algebraic groups in arbitrary characteristic, Adv. Math. 217 (2008), no. 5, 2089-2129.

[Duf13] _ Finite separating sets and quasi-affine quotients, J. Pure Appl. Algebra 217 (2013), no. 2, 247-253.

[DES14] Emilie Dufresne, Jonathan Elmer, and Müfit Sezer, Separating invariants for arbitrary linear actions of the additive group, Manuscripta Math. 143 (2014), no. 1-2, 207-219.

[DK13] Emilie Dufresne and Martin Kohls, The separating variety for the basic representations of the additive group, J. Algebra 377 (2013), 269-280.

[EK12] Jonathan Elmer and Martin Kohls, Separating invariants for the basic $\mathbb{G}_{a}$-actions, Proc. Amer. Math. Soc. 140 (2012), no. 1, 135-146.

[Har77] Robin Hartshorne, Algebraic geometry, Graduate Texts in Mathematics, No. 52, Springer-Verlag, New York, 1977.

[Igu73] Jun-ichi Igusa, Geometry of absolutely admissible representations, Number theory, algebraic geometry and commutative algebra, in honor of Yasuo Akizuki, Kinokuniya, Tokyo, 1973, pp. 373-452.

[Kem03] Gregor Kemper, Computing invariants of reductive groups in positive characteristic, Transform. Groups 8 (2003), no. 2, 159-176.

[Kra84] Hanspeter Kraft, Geometrische Methoden in der Invariantentheorie, Aspects of Mathematics, D1, Friedr. Vieweg \& Sohn, Braunschweig, 1984.

[Kru51] Wolfgang Krull, Jacobsonsche Ringe, Hilbertscher Nullstellensatz, Dimensionstheorie, Math. Z. 54 (1951), 354-387.

[Kur04] Shigeru Kuroda, A generalization of Roberts' counterexample to the fourteenth problem of Hilbert, Tohoku Math. J. (2) 56 (2004), no. 4, 501-522.

[Lun75] D. Luna, Adhérences d'orbite et invariants, Invent. Math. 29 (1975), no. 3, 231-238.

[Mat89] Hideyuki Matsumura, Commutative ring theory, second ed., Cambridge Studies in Advanced Mathematics, vol. 8, Cambridge University Press, Cambridge, 1989, Translated from the Japanese by M. Reid.

[Mum08] David Mumford, Abelian varieties, Tata Institute of Fundamental Research Studies in Mathematics, vol. 5, Published for the Tata Institute of Fundamental Research, Bombay, 2008, With appendices by C. P. Ramanujam and Yuri Manin, Corrected reprint of the second (1974) edition.

[Mum99] David Mumford, The red book of varieties and schemes, expanded ed., Lecture Notes in Mathematics, vol. 1358, Springer-Verlag, Berlin, 1999, Includes the Michigan lectures (1974) on curves and their Jacobians, With contributions by Enrico Arbarello.

[MFK94] D. Mumford, J. Fogarty, and F. Kirwan, Geometric invariant theory, third ed., Ergebnisse der Mathematik und ihrer Grenzgebiete (2) [Results in Mathematics and Related Areas (2)], vol. 34, Springer-Verlag, Berlin, 1994.

[Nag65] M. Nagata, Lectures on the fourteenth problem of Hilbert, Tata Institute of Fundamental Research, Bombay, 1965.

[RS90] Lorenzo Robbiano and Moss Sweedler, Subalgebra bases, Commutative algebra (Salvador, 1988), Lecture Notes in Math., vol. 1430, Springer, Berlin, 1990, pp. 61-87.

[Rob90] Paul Roberts, An infinitely generated symbolic blow-up in a power series ring and a new counterexample to Hilbert's fourteenth problem, J. Algebra 132 (1990), no. 2, 461-473.

[Sch68] Issai Schur, Vorlesungen über Invariantentheorie, Bearbeitet und herausgegeben von Helmut Grunsky. Die Grundlehren der mathematischen Wissenschaften, Band 143, Springer-Verlag, Berlin, 1968. 
[Slo89] Peter Slodowy, Der Scheibensatz für algebraische Transformationsgruppen, Algebraische Transformationsgruppen und Invariantentheorie, DMV Sem., vol. 13, Birkhäuser, Basel, 1989, pp. 89-113.

[Win03] Jörg Winkelmann, Invariant rings and quasiaffine quotients, Math. Z. 244 (2003), no. 1, 163-174.

[ZS60] Oscar Zariski and Pierre Samuel, Commutative algebra. Vol. II, The University Series in Higher Mathematics, D. Van Nostrand Co., Inc., Princeton, N. J.-Toronto-LondonNew York, 1960.

Department of Mathematical Sciences, Durham University, Science laboratories, South RD., Durham DH1 3LE, UK

E-mail address: e.s.dufresne@durham.ac.uk

Mathematisches Institut, Universität Basel, Rheinsprung 21, CH-4051 Basel

E-mail address: Hanspeter.Kraft@unibas.ch 